\title{
Fruitful decade of fungal metabolites as anti-diabetic agents from 2010 to 2019: emphasis on $\alpha$-glucosidase inhibitors
}

\author{
Hidayat Hussain • Mamona Nazir • Muhammad Saleem • Ahmed Al-Harrasi • \\ Elizbit • Ivan R. Green
}
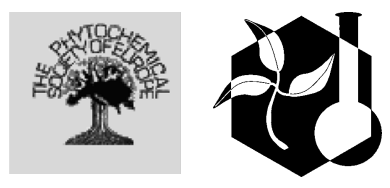

Published online: 5 February 2021

(C) The Author(s) 2021

\begin{abstract}
In recent years the prevalence of diabetes has increased globally and by 2040 the number of diabetic people has been estimated to increase to 642 million. Various classes of drugs are available to treat Type 11 diabetes. However, these drugs are associated with certain side effects. $\alpha$-Glucosidase is an intriquing target enzyme to treat Type II diabetes, and $\alpha$ glucosidase inhibitors are considered as first-line drugs for Type 11 patients. Fungi, in general, produce natural products with some amazing chemical diversity and many fungal metabolites have illustrated a wide range of biological and pharmacological effects. In this review the focus is on describing the $\alpha$ glucosidase effects and their potential as anti-diabetic agents of various metabolites isolated from fungi.
\end{abstract}

\footnotetext{
H. Hussain $(\bowtie)$

Department of Bioorganic Chemistry, Leibniz Institute of Plant Biochemistry, Weinberg 3, 06120 Halle (Saale), Germany

e-mail: hussainchem3@gmail.com
}

\section{Nazir}

Department of Chemistry, Government Sadiq College Women University Bahawalpur, Bahawalpur 63100,

Pakistan

M. Saleem ( $\square)$

Department of Chemistry, Baghdad Campus, The Islamia University of Bahawalpur, Bahawalpur 63100, Pakistan e-mail: m.saleem@iub.edu.pk
Keywords Fungi $\cdot$ Secondary metabolites $\cdot \alpha$ Glucosidase · Anti-diabetic

\section{Introduction}

Diabetes mellitus (DM) is a metabolic disorder associated with insulin resistance and the inability of the pancreatic $\beta$-cells to produce insulin, which leads to hyperglycemia. Moreover, hyperglycemia is associated with polyuria, weight loss, ketoacidosis, polydipsia, and other life-threatening health conditions (Usman et al. 2019). AGIs are an intriguing class of pharmaceutical drugs most often considered as firstline antidiabetic drugs for Type 11 patients (Hossain and Pervin 2018; Usman et al. 2019). However, in

\footnotetext{
A. Al-Harrasi

Natural and Medical Sciences Research Center,

University of Nizwa, Nizwa 616, Sultanate of Oman

Elizbit

Department Materials Engineering, National University of Sciences and Technology (NUST) H12, Islamabad, Pakistan

I. R. Green

Department of Chemistry and Polymer Science,

University of Stellenbosch,

Private Bag X1, Matieland, Stellenbosch 7600, South

Africa
} 
some studies, it has been reported that AGIs can be employed as second-line antidiabetic drugs if these inhibitors are used as combination therapy with metformin (Chan et al. 2018).

The $\alpha$-glucosidase enzyme (EC 3.2.1.20) has been considered as an important therapeutic target to treat carbohydrate mediated illnesses. It is well known that the secretion of $\alpha$-glucosidase occurs in the small intestine and this enzyme catalyzes the cleavage of disaccharides and oligosaccharides into monosaccharides in the final step of carbohydrate digestion. Moreover, the conversion of complex carbohydrates into monosaccharides enhances the glucose body level (Abbas et al. 2019). Numerous studies have demonstrated that $\alpha$-glucosidase inhibitors slow down the absorption and digestion of carbohydrates and therefore reduce the postprandial blood glucose concentrations which thus require less demand for insulin. $\alpha-$ Glucosidase inhibitors are considered mild compared to other oral antihyperglycemic agents because of their confined operation in the intestine rather than regulating various complex biochemical operations within the body (Abbas et al. 2019).

Fungi are considered one of the richest sources of natural products among living organisms because fungi have a unique metabolic system and can synthesise various types of natural products with quite intriquing chemical diversity (Srivastava 2019). After the discovery of penicillin (penicillin F) in 1929 by Alexander Fleming, substantial research on fungi lead to the isolation of thousands of new fungal metabolites with a diverse range of biological and pharmacological effects (Srivastava 2019). Besides, penicillins (antibacterial), echinocandin B (antifungal), cyclosporin A (immunosuppressive, and lovastatin (cholesterol-lowering) are all fungal originated and marketed pharmaceutical drugs. Furthermore, these factors evidently illustrate the significance of fungal metabolites to be a sustainable resource for new pharmaceutical agents.

Currently, acarbose and miglitol are two commercially available drugs with activity described as $\alpha$ glucosidase inhibitors (AGIs). These pharmaceutical drugs furthermore inhibit the absorption of carbohydrates from the gut and thus these anti-diabetic drugs are either administered alone or in combination with insulin (Bhatia et al. 2019; Hung et al. 2012). However, serious gastrointestinal damage and liver injuries have been associated with the use of these
AGIs, and this has restricted their clinical usage (Yin et al. 2014; Kao et al. 2016; Usman et al. 2019). Therefore, there is a crucial need to discover and develop new and safer anti-diabetic drugs with low toxicity. Natural products for this purpose would be ideal if they could combat such diseases without creating other secondary health issues. Plant secondary metabolites have especially been widely studied for their potential anti-diabetic properties. It is thus conceivable that diverse compounds derived from fungal sources could be developed or transformed into new therapeutics against diabetes. This review article describes some small molecules isolated in the last decade (2010 to 2019) from various fungi and developed as inhibitors of $\alpha$-glucosidase, and thus represent potential anti-diabetic drug leads.

\section{Alkaloids}

Alkaloids are nitrogen containing natural products and have been recognized substances in the treatment of human diseases for many years (Pervaiz et al. 2016; Rehman and Khan 2016). Literature indicated that a significant number of alkaloids from plant sources have been identified as $\alpha$-glucosidase inhibitors (Yin et al. 2014). On the other hand, a number of alkaloids have been reported from various fungi possessing a diverse range of biological effects (Mahmood et al. 2010). Some alkaloids have also been reported from various fungi, which illustrated $\alpha$-glucosidase inhibition. Valle et al. reported that benzomalvin A (1), quinolactacins A1 (2), A2 (3) in a mixture between B (4) and asperphenamate (5) (Fig. 1) were produced from the fungus Penicillium spathulatum (Valle et al. 2016).

Moreover, the crude extract of $P$. spathulatum displays $\alpha$-glucosidase effects with $\mathrm{IC}_{50}: 56.5 \mu \mathrm{g} / \mathrm{mL}$. Indeed, compounds $1\left(\mathrm{IC}_{50}: 383.2 \mu \mathrm{g} / \mathrm{mL}\right)$, the mixture of 2 and $\mathbf{3}\left(\mathrm{IC}_{50}: 273.3 \mu \mathrm{g} / \mathrm{mL}\right), \mathbf{4}\left(\mathrm{IC}_{50}: 57.3 \mu \mathrm{g} /\right.$ $\mathrm{mL})$, and $5\left(\mathrm{IC}_{50}: 8.3 \mu \mathrm{g} / \mathrm{mL}\right)$ display a reasonable degree of $\alpha$-glucosidase inhibition (Table 1 ). With an in vivo oral sucrose tolerance evaluation; compound $\mathbf{1}$ was also tested in normal and hyperglycemic mice $(p<0.05)$. Further docking studies revealed a higher binding affinity of $\mathbf{1}$ to yeast and mammalian $\alpha$ glucosidase and this activity has been reported as even higher than that of acarbose. The formalin assay studies substantiated antihyperalgesic activity 


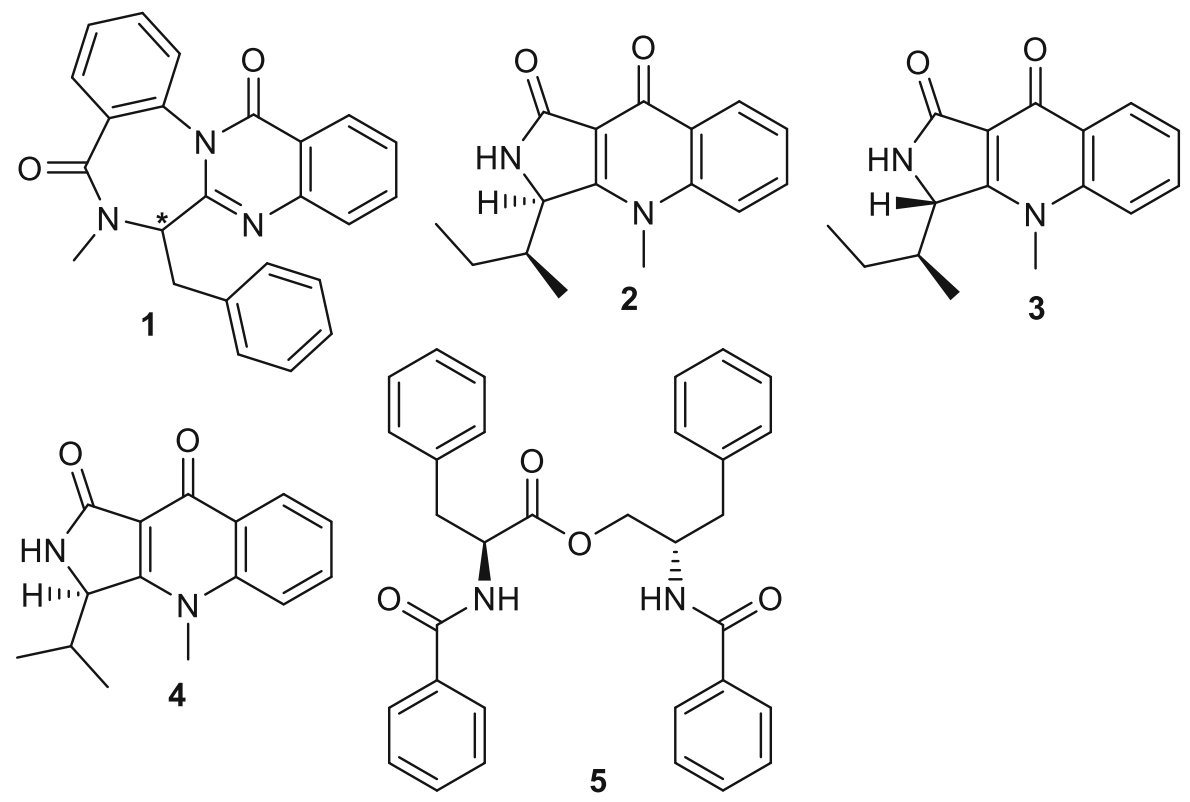

Fig. 1 Structures of alkaloids 1-5

$(p<0.05)$ of compound $\mathbf{1}$ in hyperglycemic mice (Valle et al. 2016). Benzomalvin A (1) was also previously reported from the fungus Penicillium $\mathrm{sp}$ (Sun et al. 1994), quinolactacins A1 (2), A2 (3) from the fungus Xylariaceae sp (Nong et al. 2014), and quinolactacin B (4) from the fungus Penicillium sp. (Takahashi et al. 2000; Kakinuma et al. 2000). On the other hand, asperphenamate (5) was reported as a fungal metabolite from Penicillium sp. (Frisvad et al. 2013; Arai et al. 2017) and the fungus Aspergillus versicolor (Hou et al. 2017) as well as a plant metabolite reported from Antidesma ghaesembilla (Schaefer et al. 2017) and Erythrina droogmansiana (Yaya et al. 2014).

Isoindolinone type alkaloids, viz., the sterenins A-C (6-8) (Ito-Kobayashi et al. 2008; Wang et al. 2014) and K-M (9-11) Wang et al. 2014) (Fig. 2) were reported from the fungus Stereum sp. and tested for their potential as $\alpha$-glucosidase inhibitors. The chemical diversity generated among these alkaloids is mainly due to the different substituent groups on nitrogen (Wang et al. 2014). These compounds possess $\alpha$-glucosidase effects with $\mathrm{IC}_{50}: 3.31$ to $36.6 \mu \mathrm{M}$ (Table 1). Notably, alkaloid 8 illustrated potent inhibition with $\mathrm{IC}_{50}: 3.31 \mu \mathrm{M}$. SAR studies demonstrated that the different substituents on nitrogen play a most important role and when there is no substituent $(\mathrm{R}=\mathrm{H})$ the activity was enhanced (alkaloid 8). On the other hand, various alky substituents attached to the nitrogen dramatically decreased activities (Wang et al. 2014). Penicidone C (12) was reported from Penicillium sp. (He et al. 2019; Ge et al. 2008) and demonstrated $\alpha$-glucosidase inhibition with a low $\mathrm{IC}_{50}: 51.9 \mu \mathrm{M}$ (He et al. 2019).

\section{Azaphilones}

Most of the azaphilones have been reported from two fungi families viz., Xylariaceae and Trichocomaceae and most of these compounds were reported from the fungal genera viz., Chaetomium, Penicillium, Monascus and Talaromyces. The azaphilones illustrated interesting pharmacological effects viz., anti-fungal, antimicrobial, antioxidant, antiviral, anti-inflammatory, cytotoxic, and nematicidaland activities (Osmanova et al. 2010; Gao et al. 2013). Talaraculones A (13) and B (14) (Fig. 3) were reported from the fungus Talaromyces aculeatus and compounds 13 and 14 illustrated $\alpha$-glucosidase potential with $\mathrm{IC}_{50}: 78.6$ and $22.9 \mu \mathrm{M}$, respectively, which are lower than acarbose $\left(\mathrm{IC}_{50}: 101.5 \mu \mathrm{M}\right)($ Ren et al. 2017).

Chermesinone A (15), isolated from the fungus Penicillium chermesinum, illustrated $\alpha$-glucosidase effects with $\mathrm{IC}_{50}: 24.5 \mu \mathrm{M}$ (Huang et al. 2011). In this regard, compound $\mathbf{1 5}$ was also reported from the 
Table 1 Fungal metabolites $\mathbf{1 - 3 9}$ as $\boldsymbol{\alpha}$-glucosidase inhibitors

\begin{tabular}{|c|c|c|c|}
\hline Compound & Source & $\begin{array}{l}\alpha \text {-Glucosidase } \\
\text { activity }\end{array}$ & References \\
\hline Benzomalvin A (1) & Penicillium spathulatum & $\mathrm{IC}_{50}: 383.2 \mu \mathrm{M}$ & Valle et al. (2016) \\
\hline Quinolactacins A1 (2), A2 (3); mixture & Penicillium spathulatum & $\mathrm{IC}_{50}: 273.3 \mu \mathrm{M}$ & Valle et al. (2016) \\
\hline Benzomalvin B (4) & Penicillium spathulatum & $\mathrm{IC}_{50}: 57.3 \mu \mathrm{M}$ & Valle et al. (2016) \\
\hline Asperphenamate (5) & Penicillium spathulatum & $\mathrm{IC}_{50}: 8.3 \mu \mathrm{M}$ & Valle et al. (2016) \\
\hline Sterenin A $(\mathbf{6})$ & Stereum hirsutum & $\mathrm{IC}_{50}: 25.1 \mu \mathrm{M}$ & Wang et al. (2014) \\
\hline Sterenin B (7) & Stereum hirsutum & $\mathrm{IC}_{50}: 12.3 \mu \mathrm{M}$ & Wang et al. (2014) \\
\hline Sterenin C (8) & Stereum hirsutum & $\mathrm{IC}_{50}: 3.3 \mu \mathrm{M}$ & Wang et al. (2014) \\
\hline Sterenin K (9) & Stereum hirsutum & $\mathrm{IC}_{50}: 36.6 \mu \mathrm{M}$ & Wang et al. (2014) \\
\hline Sterenin L (10) & Stereum hirsutum & $\mathrm{IC}_{50}: 13.0 \mu \mathrm{M}$ & Wang et al. (2014) \\
\hline Sterenin M (11) & Stereum hirsutum & $\mathrm{IC}_{50}: 27.5 \mu \mathrm{M}$ & Wang et al. (2014) \\
\hline Penicidone C (12) & Penicillium sp. & $\mathrm{IC}_{50}: 51.9 \mu \mathrm{M}$ & He et al. (2019) \\
\hline Talaraculone A (13) & Talaromyces aculeatus & $\mathrm{IC}_{50}: 78.6 \mu \mathrm{M}$ & Ren et al. (2017) \\
\hline Talaraculone B (14) & Talaromyces aculeatus & $\mathrm{IC}_{50}: 22.9 \mu \mathrm{M}$ & Ren et al. (2017) \\
\hline Chermesinone A (15) & $\begin{array}{l}\text { Penicillium } \\
\text { chermesinum }\end{array}$ & $\mathrm{IC}_{50}: 24.5 \mu \mathrm{M}$ & Huang et al. (2011) \\
\hline Pinazaphilone A (16:) & Penicillium sp. & $\mathrm{IC}_{50}: 81.7 \mu \mathrm{M}$ & Liu et al. (2015) \\
\hline Pinazaphilone B (17) & Penicillium sp. & $\mathrm{IC}_{50}: 28.0 \mu \mathrm{M}$ & Liu et al. (2015) \\
\hline Sch $1385568(\mathbf{1 8})$ & Penicillium sp. & $\mathrm{IC}_{50}: 16.6 \mu \mathrm{M}$ & Liu et al. (2015) \\
\hline Sch 725680 (19) & Penicillium sp. & $\mathrm{IC}_{50}: 33.8 \mu \mathrm{M}$ & He et al. (2019) \\
\hline 6'-O-desmethylterphenyllin (20) & $\begin{array}{l}\text { Penicillium } \\
\text { chermesinum }\end{array}$ & $\mathrm{IC}_{50}: 2.5 \mu \mathrm{M}$ & Huang et al. (2011) \\
\hline 3-hydroxy-6'-O-desmethylterphenyllin (21) & $\begin{array}{l}\text { Penicillium } \\
\text { chermesinum }\end{array}$ & $\mathrm{IC}_{50}: 4.9 \mu \mathrm{M}$ & Huang et al. (2011) \\
\hline 3,3"-dihydroxy-6'-O-desmethylterphenyllin (22) & $\begin{array}{l}\text { Penicillium } \\
\text { chermesinum }\end{array}$ & $\mathrm{IC}_{50}: 0.9 \mu \mathrm{M}$ & Huang et al. (2011) \\
\hline Sarcoviolin $\beta$ (23) & Sarcodon leucopus & $\mathrm{IC}_{50}: 0.58 \mu \mathrm{M}$ & Ma et al. (2014) \\
\hline Episarcoviolin $\beta$ (24) & Sarcodon leucopus & $\mathrm{IC}_{50}: 1.07 \mu \mathrm{M}$ & Ma et al. (2014) \\
\hline $2^{\prime}, 3^{\prime}, 5^{\prime}, 6^{\prime}$-Tetracetoxy-4,4" -dihydroxy-p-terphenyl (25) & Sarcodon leucopus & $\mathrm{IC}_{50}: 35 \mu \mathrm{M}$ & Ma et al. (2014) \\
\hline $2^{\prime}, 3^{\prime}$-Diacetoxy-4,4" $, 5^{\prime}, 6^{\prime}$-tetrahydroxy-p-terphenyl (26) & Sarcodon leucopus & $\mathrm{IC}_{50}: 19 \mu \mathrm{M}$ & Ma et al. (2014) \\
\hline $\begin{array}{l}2^{\prime}, 3^{\prime} \text {-Diacetoxy-3,4,4" }, 5^{\prime}, 6^{\prime} \text {-pentahydroxy-p-terphenyl } \\
\quad(\mathbf{2 7})\end{array}$ & Sarcodon leucopus & $\mathrm{IC}_{50}: 3.3 \mu \mathrm{M}$ & Ma et al. (2014) \\
\hline Leucomelone (28) & Sarcodon leucopus & $\mathrm{IC}_{50}: 3.5 \mu \mathrm{M}$ & Ma et al. (2014) \\
\hline B1-V (29) & Sarcodon lеисориs & $\mathrm{IC}_{50}: 6.2 \mu \mathrm{M}$ & Ma et al. (2014) \\
\hline Episarcodonin $\alpha(\mathbf{3 0})$ & Sarcodon leucopus & $\mathrm{IC}_{50}: 3.6 \mu \mathrm{M}$ & Ma et al. (2014) \\
\hline Episarcodonin (31) & Sarcodon lеисориs & $\mathrm{IC}_{50}: 4.2 \mu \mathrm{M}$ & Ma et al. (2014) \\
\hline Sarcodonin $\alpha(32)$ & Sarcodon leucopus & $\mathrm{IC}_{50}: 1.2 \mu \mathrm{M}$ & Ma et al. (2014) \\
\hline Concrescenin A (33) & Hydnellum concrescens & $\mathrm{IC}_{50}: 0.9 \mu \mathrm{M}$ & Wang et al. (2014a) \\
\hline Concrescenin B (34) & Hydnellum concrescens & $\mathrm{IC}_{50}: 3.1 \mu \mathrm{M}$ & Wang et al. (2014a) \\
\hline Thelephantin L (35) & Hydnellum concrescens & $\mathrm{IC}_{50}: 4.5 \mu \mathrm{M}$ & Wang et al. (2014a) \\
\hline Thelephantin I (36) & Hydnellum concrescens & $\mathrm{IC}_{50}: 18.7 \mu \mathrm{M}$ & Wang et al. (2014a) \\
\hline Thelephantin K (37) & Hydnellum concrescens & $\mathrm{IC}_{50}: 2.9 \mu \mathrm{M}$ & Wang et al. (2014a) \\
\hline Dihydroauran-tiacin dibenzoate (3) & Hydnellum concrescens & $\mathrm{IC}_{50}: 5.1 \mu \mathrm{M}$ & Wang et al. (2014a) \\
\hline Curtisian A (39) & Hydnellum concrescens & $\mathrm{IC}_{50}: 8.3 \mu \mathrm{M}$ & Wang et al. (2014a) \\
\hline
\end{tabular}


Fig. 2 Structures of alkaloids 6-12<smiles>[R]CN1C(=O)c2cc(OC(=O)c3c(C)cc(O)cc3O)c(CC=C(C)C)c(O)c21</smiles><smiles>[R]C(C(=O)O)N1Cc2c(cc(OC(=O)c3c(C)cc(O)cc3O)c(CC=C(C)C)c2O)C1=O</smiles>

6: $\mathrm{R}=\left(\mathrm{CH}_{2}\right)_{2} \mathrm{OH}$

8: $\mathrm{R}=\mathrm{H}$

9: $\mathrm{R}=\left(\mathrm{CH}_{2}\right)_{3} \mathrm{OH}$
7: $\mathrm{R}=\left(\mathrm{CH}_{2}\right)_{2} \mathrm{OH}$

11: $\mathrm{R}=\mathrm{CH}_{2} \mathrm{CH}(\mathrm{Me})_{2}$<smiles>C/C=C/c1cc(=O)c(C(=O)c2c(OC)cc(OC)cc2C(=O)OC)c[nH]1</smiles>

10

fungus Phomopsis sp. (Yang et al. 2015). Pinazaphilones A (16: $\left.\mathrm{IC}_{50}: 81.7 \mu \mathrm{M}\right)$ and $\mathrm{B}\left(17: \mathrm{IC}_{50}\right.$ : $28.0 \mu \mathrm{M})$, and Sch $1385568\left(\mathbf{1 8}\right.$ IC $\left._{50}: 16.6 \mu \mathrm{M}\right)$ were produced by the fungus Penicillium sp. and illustrated good to moderate $\alpha$-glucosidase effects (Table 1) (Liu et al. 2015a). Sch 725680 (19) was reported from Penicillium sp. (He et al. 2019) and was shown to possess $\alpha$-glucosidase effects with $\mathrm{IC}_{50}: 33.8 \mu \mathrm{M}$.

\section{p-Terphenyls}

p-Terphenyls bearing a C-18 tricyclic or polycyclic aromatic core demonstrate a huge chemical diversity generated among these compounds by suitable changes in the middle aromatic ring or the linkages between the rings ( $\mathrm{Li}$ et al. 2018). Over 230 p-terphenyl analogs have been reported as well as a number of isolated derivatives ( $\mathrm{Li}$ et al. 2018). The majority of these compounds were reported from fungi ( $\mathrm{Li}$ et al. 2018; Quang et al. 2003; Liu et al. 2004; Lee et al. 1996; Nagasawa et al. 2014). The three $p$-terphenyls 20-22 (Fig. 4) were isolated from the fungus Penicillium chermesinum and illustrated $\alpha$-glucosidase effects with $\mathrm{IC}_{50}$ values of $2.5,4.9$, and $0.9 \mu \mathrm{M}$, respectively (Table 1). Notably, the activity of these compounds was higher than the reference genistein $\left(\mathrm{IC}_{50}: 9.8 \mu \mathrm{M}\right.$, Huang et al. 2011). Previously, compound 20 was reported from the fungus Penicillium raistrickii (Belofsky et al. 1998).

p-Terphenyls 23-32 were produced by the fungus Sarcodon leucopus and their structures were determined via extensive NMR techniques (Ma et al. 2014). All compounds displayed $\alpha$-glucosidase effects with 
<smiles>[R4]C=[R4]C=CC(=O)C=C[C@@H](C)O</smiles><smiles>CC[C@H](C)C(=O)C[C@]1(C(C)C)C2=COC(C)=CC2=CC(=O)[C@]1(C)O</smiles><smiles>[Y16][C@]1(O)C(=O)C=C2C=C(/C=C/C(=O)O)OC[C@H]2[C@H]1OC(=O)c1c(C)cc(O)cc1O</smiles><smiles>C/C=C/C1=CC2=CC(=O)[C@](C)(O)[C@H](OC(=O)c3c(C)cc(O)cc3O)C2=CO1</smiles><smiles>Cc1cc(O)cc(O)c1C(=O)O</smiles><smiles>C/C=C/C1=CC2=CC(=O)[C@](C)(O)[C@H](O)[C@H]2CO1</smiles>

Fig. 3 Structures of azaphilones 13-19

$\mathrm{IC}_{50}$ values ranging from 0.58 to $35.0 \mu \mathrm{M}$. Among these, sarcoviolin $\beta$ (23) showed good and potent effects with an $\mathrm{IC}_{50}: 0.58 \mu \mathrm{M}$ followed by an isomer of 24. Compounds 24 and 27-32 illustrated moderate inhibition with $\mathrm{IC}_{50}$ values ranging from 1 to $10 \mu \mathrm{M}$ (Table 1). On the other hand p-terphenyls 25 and $\mathbf{2 6}$ possess relatively weak effects with $\mathrm{IC}_{50}$ values of 35.0 and $19.0 \mu \mathrm{M}$, respectively. An SAR analysis demonstrated that the configuration at $\mathrm{N}-1 \beta$ and $\mathrm{C}-2 \beta$ greatly effects the $\alpha$-glucosidase activity. For instance, metabolites 23 and $\mathbf{3 2}$ having the cis $\mathrm{N}-1 \beta$ and $\mathrm{C}-2 \beta$ displayed better activity than metabolites $\mathbf{2 4}$ and $\mathbf{3 0}$ bearing a trans configuration (Ma et al. 2014).

p-Terphenyl analogs viz., concrescenins A (33) and B (34), thelephantins L (35), I (36), K (37), compound 38, and curtisian A (39) (Fig. 5) were reported from the fungus Hydnellum concrescens (Wang et al. 2014a). p-Terphenyl analogs 33-39 illustrated $\alpha$ glucosidase effects with the $\mathrm{IC}_{50}$ ranging from of 0.99 to $18.77 \mu \mathrm{M}$. Among the tested compounds, metabolite $33\left(\mathrm{IC}_{50}: 0.99 \mu \mathrm{M}\right)$ possesses the strongest effects followed by metabolite $34\left(\mathrm{IC}_{50}: 3.11 \mu \mathrm{M}\right)$ and
$37\left(\mathrm{IC}_{50}: 2.98 \mu \mathrm{M}\right)$ (Table 1). A preliminary SAR study demonstrated that the benzene core in the center of the $p$-terphenyl core enhances the $\alpha$-glucosidase effects when compared to the benzoquinone ring as can be noticed by metabolites 33-35 and 37-39 displaying better activity than compound $\mathbf{3 6}$ (Wang et al. 2014a).

\section{Depsides}

The fungus MEXU 27095 produces the tridepsides, thielavins A (40), J (41) and K (42) (Fig. 6). Moreover, the activities of thielavins $\mathrm{A}\left(\mathbf{4 0}: \mathrm{IC}_{50}: 23.8 \mu \mathrm{M} ; \mathrm{K}_{\mathrm{i}}\right.$ : $27.8 \mu \mathrm{M}), \mathrm{J}\left(\mathbf{4 1}: \mathrm{IC}_{50}: 15.8 \mu \mathrm{M} ; \mathrm{K}_{\mathrm{i}}: 66.2 \mu \mathrm{M}\right)$, and $\mathrm{K}$ (42: $\left.\mathrm{IC}_{50}: 22.1 \mu \mathrm{M} ; \mathrm{K}_{\mathrm{i}}: 22.1 \mu \mathrm{M}\right)$ illustrated good Saccharomyces cerevisieae a-glucosidase inhibition (Table 2). Notably, the activities of these compounds were higher than that of acarbose $\left(\mathrm{IC}_{50}: 545 \mu \mathrm{M}\right)$. Metabolites 40-42 are reported as being non-competitive inhibitors with $\mathrm{K}_{\mathrm{i}}$ values ranging from 27.8 to $66.2 \mu \mathrm{M}$. Thielavin $\mathrm{J}\left(\mathbf{4 1}: \mathrm{IC}_{50}: 30.5 \mu \mathrm{M}\right)$ also 


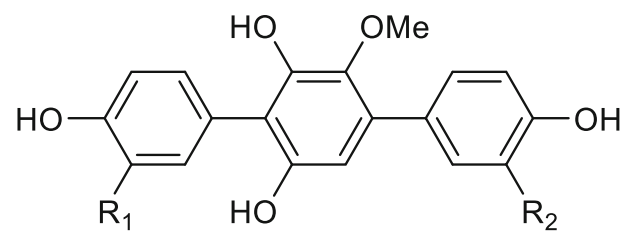

20: $\mathrm{R}_{1}=\mathrm{R}_{2}=\mathrm{OH}$

21: $\mathrm{R}_{1}=\mathrm{OH}, \mathrm{R}_{2}=\mathrm{H}$<smiles>CC[C@H](C)C1=C(O)N(O)C(=O)[C@@]2([C@@H](C)CC)Oc3cc(C4=C(O)C(=O)C(c5ccc(O)cc5)=C(O)C4=O)ccc3O[N+]12[O-]</smiles>

22: $R_{1}=R_{2}=H$

23

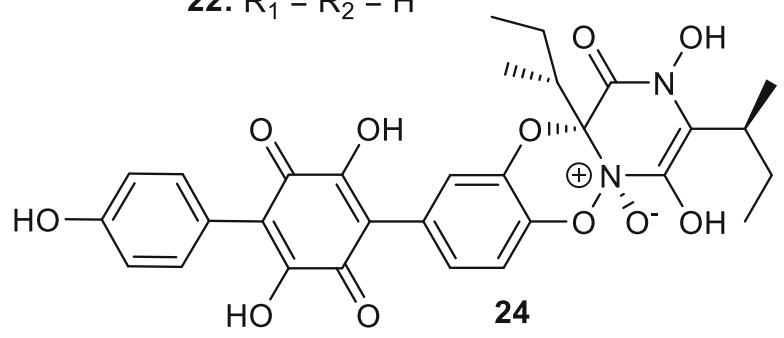

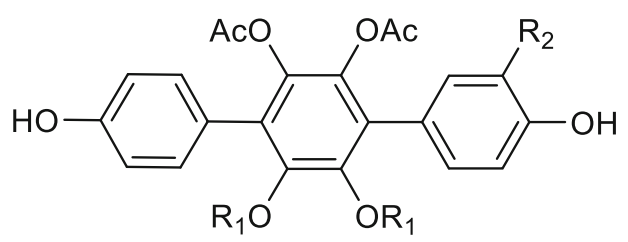

25: $R_{1}=A c, R_{2}=H$

26: $R_{1}=R_{2}=H$

27: $\mathrm{R}_{1}=\mathrm{H}, \mathrm{R}_{2}=\mathrm{OH}$<smiles>O=C1C(O)=C(c2ccc(O)c(O)c2)C(=[123I])C(O)=C1c1ccc(O)cc1</smiles>

28

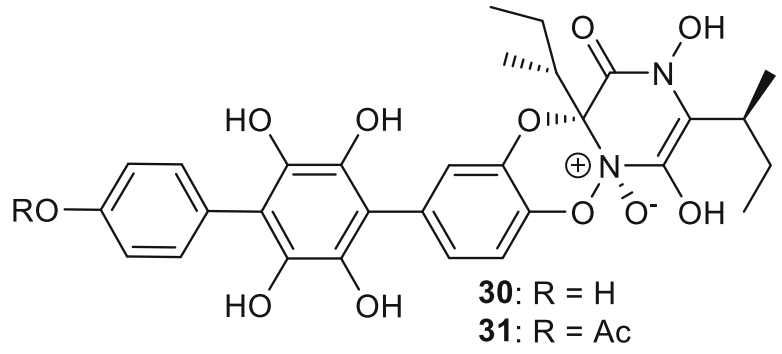

Fig. 4 Structures of p-terphenyls 20-32

inhibited the effects of Bacillus stearothermophilusbased $\alpha$-glucosidase (Rivera-Chávez et al. 2013).

Sterenins E-J (43-48), MS-13 (49) and 50 were reported from Stereum hirsutum and these compounds were all shown to possess $\alpha$-glucosidase effects with $\mathrm{IC}_{50}$ : 3.06-72.50 $\mu \mathrm{M}$ (Table 2). Compounds $\mathbf{4 3 - 4 5}$ $\left(\mathrm{IC}_{50}\right.$ : 3.06 to $\left.7.62 \mu \mathrm{M}\right)$ illustrated higher activities than compounds 46-50 indicating that the ring $\mathrm{B}$ carbonyl moiety substitution can enhance the activity. Moreover, compound $\mathbf{4 3}$ has much stronger effects than metabolite $\mathbf{4 8}$ and this finding confirmed that the isoprenyl group significantly affects the inhibitory activity (Wang et al. 2014). Colletotric A (51: IC $_{50}$ :
$36.2 \mu \mathrm{M}), \mathrm{B}\left(\mathbf{5 2}: \mathrm{IC}_{50}: 35.8 \mu \mathrm{M}\right)$, and $\mathrm{C}\left(\mathbf{5 3}: \mathrm{IC}_{50}\right.$ : $60.2 \mu \mathrm{M})$ were reported from the fungus Phoma sp. and showed $\alpha$-glucosidase effects (Chen et al. 2019) (Table 2).

\section{Depsidones}

The depsidone talaromyone B (54) was reported from the fungus Talaromyces stipitatus (Cai et al. 2017) while purpactin A (55) was obtained from the fungi $T$. stipitatus (Cai et al. 2017) and Penicillium sp. (Tomoda et al. 1991; Nishida et al. 1991). In addition, 
Fig. 5 Structures of p-terphenyls 33-39<smiles>[R20]Oc1c(OC(=O)OC([R2])([R])[R])c(-c2ccc(O)cc2)c([R6])c([R20])c1-c1ccc(O)cc1</smiles>

33: $R_{1}=A c, R_{2}=R_{3}=H$

34: $R_{1}=R_{3}=A c, R_{2}=C O B z$

35: $R_{1}=R_{2}=R_{3}=C O B z$

37: $R_{1}=R_{3}=H, R_{2}=C O B z$

38: $R_{3}=H, R_{1}=R_{2}=C O B z$

39: $R_{1}=R_{2}=R_{3}=A c$<smiles>[R7]OC1=C(c2ccc(O)cc2)C(=O)C(OC)=C(c2ccc(O)cc2)C1=O</smiles>

36<smiles>[R2]c1c(C)c(C(=O)O)c(O)c(C)c1OC(=O)c1c(C)c(C)c(OC(=O)c2c(C)cc(O)c(C)c2O)c(C)c1C</smiles><smiles>[R1]c1cc(OC(=O)c2c(C)cc(O)cc2O)c(CC=C(C)C)c([R2])c1[R2]</smiles>

40: $\mathrm{R}_{1}=\mathrm{H}, \mathrm{R}_{2}=\mathrm{Me} \quad$ 43: $\mathrm{R}_{1}=\mathrm{Me}, \mathrm{R}_{2}=\mathrm{CO}_{2} \mathrm{H}, \mathrm{R}_{3}=\mathrm{H}$

41: $R_{1}=M e, R_{2}=H \quad$ 44: $R_{1}=M e, R_{2}=C_{2} M e, R_{3}=H$

42: $R_{1}=R_{2}=M e$

45: $\mathrm{R}_{1}=\mathrm{Me}, \mathrm{R}_{2}=\mathrm{CHO}, \mathrm{R}_{3}=\mathrm{H}$<smiles>Cc1cc(OC(=O)c2c(C)cc(O)cc2O)cc2c1O[C@H](C(C)(C)O)C2</smiles>

46: $\mathrm{R}_{1}=\mathrm{CH}_{2} \mathrm{OH}, \mathrm{R}_{2}=\mathrm{CH}_{2} \mathrm{OMe}, \mathrm{R}_{3}=\mathrm{H}$

47: $\mathrm{R}_{1}=\mathrm{CH}_{2} \mathrm{OH}, \mathrm{R}_{2}=\mathrm{CH}_{2} \mathrm{OCH}_{2} \mathrm{CH}(\mathrm{OH}) \mathrm{CH}_{2} \mathrm{OAc}, \mathrm{R}_{3}=\mathrm{H}$

49: $\mathrm{R}_{1}=\mathrm{R}_{2}=\mathrm{CH}_{2} \mathrm{OH}, \mathrm{R}_{3}=\mathrm{H}$

50: $\mathrm{R}_{1}=\mathrm{H}, \mathrm{R}_{2}=\mathrm{OH}, \mathrm{R}_{3}=\mathrm{OMe}$<smiles>COc1c(C)c(C)c(C(=O)Oc2cc(O)c(C(C)=O)c(C)c2C)c(C)c1O</smiles><smiles>[R]C(=O)c1c(O)cc(OC(=O)c2c(C)c(OC)c(C)c(OC(=O)c3c(C)cc(O)cc3O)c2C)c(C)c1C</smiles>

52: $\mathrm{R}=\mathrm{OMe}$

Fig. 6 Structures of p-terphenyls 40-53

tenellic acid A (56) (Fig. 7) was isolated from the fungi T. stipitatus (Cai et al. 2017) and Camposporium quercicola (Wang et al. 2008) and its structure was established via NMR spectroscopic methods as well as employing the Mosher's protocol. Compounds 54-56 illustrated moderate $\alpha$-glucosidase activity (Table 2) 
Table 2 Fungal metabolites $\mathbf{4 0 - 7 3}$ as $\alpha$-glucosidase inhibitors

\begin{tabular}{|c|c|c|c|}
\hline Compd. & Source & $\begin{array}{l}\text { A-Glucosidase } \\
\text { activity }\end{array}$ & References \\
\hline Thielavin A (40) & $\begin{array}{l}\text { Fungus MEXU } \\
27095\end{array}$ & $\begin{array}{l}\mathrm{IC}_{50}: 23.8 \mu \mathrm{M} ; \mathrm{K}_{\mathrm{i}} \\
\quad 27.8 \mu \mathrm{M}\end{array}$ & $\begin{array}{l}\text { Rivera-Chávez } \\
\text { et al. (2013) }\end{array}$ \\
\hline Thielavin J (41) & $\begin{array}{l}\text { Fungus MEXU } \\
27095\end{array}$ & $\begin{array}{l}\mathrm{IC}_{50}: 15.8 ; \mathrm{K}_{\mathrm{i}}: \\
\quad 66.2 \mu \mathrm{M}\end{array}$ & $\begin{array}{l}\text { Rivera-Chávez } \\
\text { et al. (2013) }\end{array}$ \\
\hline Thielavin K (42) & $\begin{array}{l}\text { Fungsu MEXU } \\
27095\end{array}$ & $\begin{array}{l}\mathrm{IC}_{50}: 22.1 \mu \mathrm{M} ; \mathrm{K}_{\mathrm{i}}: \\
\quad 22.1 \mu \mathrm{M}\end{array}$ & $\begin{array}{l}\text { Rivera-Chávez } \\
\text { et al. (2013) }\end{array}$ \\
\hline Sterenin E (43) & Stereum hirsutum & $\mathrm{IC}_{50}: 7.6 \mu \mathrm{M}$ & Wang et al. (2014) \\
\hline Sterenin F (44) & Stereum hirsutum & $\mathrm{IC}_{50}: 3.0 \mu \mathrm{M}$ & Wang et al. (2014) \\
\hline Sterenin G (45) & Stereum hirsutum & $\mathrm{IC}_{50}: 6.0 \mu \mathrm{M}$ & Wang et al. (2014) \\
\hline Sterenin H (46) & Stereum hirsutum & $\mathrm{IC}_{50}: 22.7 \mu \mathrm{M}$ & Wang et al. (2014) \\
\hline Sterenin I (47) & Stereum hirsutum & $\mathrm{IC}_{50}: 72.5 \mu \mathrm{M}$ & Wang et al. (2014) \\
\hline Sterenin $\mathrm{J}(\mathbf{4 8})$ & Stereum hirsutum & $\mathrm{IC}_{50}: 65.7 \mu \mathrm{M}$ & Wang et al. (2014) \\
\hline MS-13 (49) & Stereum hirsutum & $\mathrm{IC}_{50}: 23.8 \mu \mathrm{M}$ & Wang et al. (2014) \\
\hline $\begin{array}{l}\text { 4-Hydroxy-3-methoxy-2-(3-methylbut-2-en-1-yl)phenyl 2,4- } \\
\text { dihydroxy-6-methylbenzoate (50) }\end{array}$ & Stereum hirsutum & $\mathrm{IC}_{50}: 14.7 \mu \mathrm{M}$ & Wang et al. (2014) \\
\hline Colletotric A (51) & Phoma sp. & $\mathrm{IC}_{50}: 36.2 \mu \mathrm{M}$ & Chen et al. (2019) \\
\hline Colletotric B (52) & Phoma sp. & $\mathrm{IC}_{50}: 35.8 \mu \mathrm{M}$ & Chen et al. (2019) \\
\hline Colletotric C (53) & Phoma sp. & $\mathrm{IC}_{50}: 60.2 \mu \mathrm{M}$ & Chen et al. (2019) \\
\hline Talaromyone B (54) & $\begin{array}{l}\text { Talaromyces } \\
\text { stipitatus }\end{array}$ & $\mathrm{IC}_{50}: 48.4 \mu \mathrm{M}$ & Cai et al. (2017) \\
\hline Purpactin A (55) & $\begin{array}{l}\text { Talaromyces } \\
\text { stipitatus }\end{array}$ & $\mathrm{IC}_{50}: 80.9 \mu \mathrm{M}$ & Cai et al. (2017) \\
\hline Tenellic acid A (56) & $\begin{array}{l}\text { Talaromyces } \\
\text { stipitatus }\end{array}$ & $\mathrm{IC}_{50}: 99.8 \mu \mathrm{M}$ & Cai et al. (2017) \\
\hline Botryorhodine E (57) & $\begin{array}{l}\text { Meyerozyma } \\
\text { guilliermondii }\end{array}$ & $\mathrm{IC}_{50}: 15.4 \mu \mathrm{M}$ & Chen et al. (2015a) \\
\hline Botryorhodine F (58) & $\begin{array}{l}\text { Meyerozyma } \\
\quad \text { guilliermondii }\end{array}$ & $\mathrm{IC}_{50}: 9.8 \mu \mathrm{M}$ & Chen et al. (2015a) \\
\hline \multirow[t]{2}{*}{ Botryorhodine G (59) } & $\begin{array}{l}\text { Meyerozyma } \\
\text { guilliermondii }\end{array}$ & $\mathrm{IC}_{50}: 12.4 \mu \mathrm{M}$ & Chen et al. (2015a) \\
\hline & Trichoderma sp. & $\mathrm{IC}_{50}: 54.1 \mu \mathrm{M}$ & Zhang et al. (2017) \\
\hline Botryorhodine A (60) & $\begin{array}{l}\text { Meyerozyma } \\
\text { guilliermondii }\end{array}$ & $\mathrm{IC}_{50}: 13.3 \mu \mathrm{M}$ & Chen et al. (2015a) \\
\hline Botryorhodine B (61) & $\begin{array}{l}\text { Meyerozyma } \\
\text { guilliermondii }\end{array}$ & $\mathrm{IC}_{50}: 11.7 \mu \mathrm{M}$ & Chen et al. (2015a) \\
\hline \multirow[t]{2}{*}{ Botryorhodine D (62) } & $\begin{array}{l}\text { Meyerozyma } \\
\text { guilliermondii }\end{array}$ & $\mathrm{IC}_{50}: 2.1 \mu \mathrm{M}$ & Chen et al. (2015a) \\
\hline & Trichoderma sp. & $\mathrm{IC}_{50}: 10.3 \mu \mathrm{M}$ & Zhang et al. (2017) \\
\hline Botryorhodine H (63) & Trichoderma sp. & $\mathrm{IC}_{50}: 8.1 \mu \mathrm{M}$ & Zhang et al. (2017) \\
\hline Botryorhodine C (64) & Trichoderma sp. & $\mathrm{IC}_{50}: 11.2 \mu \mathrm{M}$ & Zhang et al. (2017) \\
\hline Compound 65 & $\begin{array}{l}\text { Talaromyces } \\
\text { amestolkiae }\end{array}$ & $\mathrm{IC}_{50}: 140.8 \mu \mathrm{M}$ & Chen et al. (2016) \\
\hline Compound 66 & $\begin{array}{l}\text { Talaromyces } \\
\text { amestolkiae }\end{array}$ & $\mathrm{IC}_{50}: 89.4 \mu \mathrm{M}$ & Chen et al. (2016) \\
\hline Compound 67 & $\begin{array}{l}\text { Talaromyces } \\
\text { amestolkiae }\end{array}$ & $\mathrm{IC}_{50}: 585.7 \mu \mathrm{M}$ & Chen et al. (2016) \\
\hline Compound 68 & $\begin{array}{l}\text { Talaromyces } \\
\text { amestolkiae }\end{array}$ & $\mathrm{IC}_{50}: 573.3 \mu \mathrm{M}$ & Chen et al. (2016) \\
\hline
\end{tabular}


Table 2 continued

\begin{tabular}{|c|c|c|c|}
\hline Compd. & Source & $\begin{array}{l}\text { A-Glucosidase } \\
\text { activity }\end{array}$ & References \\
\hline S-(-)-5,6,8-trihydroxy-4-(1'-hydroxyethyl)isocoumarin (69) & $\begin{array}{l}\text { Talaromyces } \\
\text { amestolkiae }\end{array}$ & $\mathrm{IC}_{50}: 315.3 \mu \mathrm{M}$ & Chen et al. (2016) \\
\hline Sescandelin B (70) & $\begin{array}{l}\text { Talaromyces } \\
\text { amestolkiae }\end{array}$ & $\mathrm{IC}_{50}: 17.2 \mu \mathrm{M}$ & Chen et al. (2016) \\
\hline $\begin{array}{l}\text { 6-Hydroxy-4-hydroxymethyl-8-methoxy-3-methyl-isocou-marin } \\
\text { (71) }\end{array}$ & $\begin{array}{l}\text { Talaromyces } \\
\text { amestolkiae }\end{array}$ & $\mathrm{IC}_{50}: 302.6 \mu \mathrm{M}$ & Chen et al. (2016) \\
\hline 3,4-Dimethyl-6,8-dihydroxyisocoumarin (72) & $\begin{array}{l}\text { Talaromyces } \\
\text { amestolkiae }\end{array}$ & $\mathrm{IC}_{50}: 36.4 \mu \mathrm{M}$ & Chen et al. (2016) \\
\hline 28 sescandelin $(73)$ & $\begin{array}{l}\text { Talaromyces } \\
\text { amestolkiae }\end{array}$ & $\mathrm{IC}_{50}: 417.8 \mu \mathrm{M}$ & Chen et al. (2016) \\
\hline
\end{tabular}

with $\mathrm{IC}_{50}$ values ranging from 48.4 to $99.8 \mu \mathrm{M}$ (Cai et al. 2017).

Six further depsidones viz., botryorhodines $\mathrm{E}-\mathrm{G}$ (57-59), botryorhodine A (60), B (61), D (62) were reported from the fungus Meyerozyma guilliermondii and all illustrated significant $\alpha$-glucosidase potentials with $\mathrm{IC}_{50}$ : ranging from 2.1 to $15.4 \mu \mathrm{M}$ (Table 2). Compound 62 was the most active with an $\mathrm{IC}_{50}$ value of $2.1 \mu \mathrm{M}$ followed by compounds 58, 61 and 59, which is significantly lower than that of acarbose $\left(\mathrm{IC}_{50}=553.7 \mu \mathrm{M}\right)$. An SAR analysis suggests that the hydroxymethyl group at C-3 increases the $\alpha$-glucosidase effects, whereas, the presence of a methyl group at $\mathrm{C}-3^{\prime}$ exerts no additional effect on the $\alpha$-glucosidase inhibitory power of compounds 57-62 (Chen et al. 2015a).<smiles>COc1c([C@H](CC(C)C)OC(C)=O)ccc2c1C(=O)Oc1cc(C)cc(CO)c1O2</smiles><smiles>COc1c([C@H](CC(C)C)OC(C)=O)ccc2c1C(=O)Oc1cc(C)cc(O)c1O2</smiles><smiles>COc1c([C@@H](CC(C)C)OC)ccc(Oc2c(O)cc(C)cc2C=O)c1C(=O)O</smiles>
56<smiles>[R2]c1c(O)cc(C)c2c1OC(=O)c1c(C)cc(O)c([R2])c1O2</smiles>

57: $\mathrm{R}_{1}=\mathrm{OH}, \mathrm{R}_{2}=\mathrm{H}$

58: $\mathrm{R}_{1}=\mathrm{OH}, \mathrm{R}_{2}=\mathrm{Me}$

59: $\mathrm{R}_{1}=\mathrm{CH}_{2} \mathrm{OMe}, \mathrm{R}_{2}=\mathrm{H}$

60: $\mathrm{R}_{1}=\mathrm{CHO}, \mathrm{R}_{2}=\mathrm{H}$

61: $\mathrm{R}_{1}=\mathrm{CH}_{2} \mathrm{OH}, \mathrm{R}_{2}=\mathrm{Me}$

62: $\mathrm{R}_{1}=\mathrm{CH}_{2} \mathrm{OH}, \mathrm{R}_{2}=\mathrm{H}$

Fig. 7 Structures of depsodones 54-62 


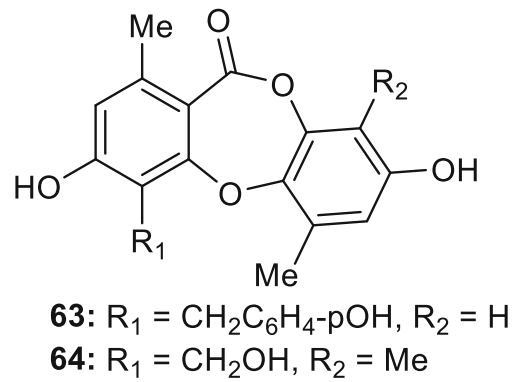

Fig. 8 Structures of depsidones 63 and 64

Another fungus Trichoderma sp. produces botryorhodines $\mathrm{G}$ (59) and $\mathrm{H}$ (63) (Fig. 8) and compound 59 possess moderate $\alpha$-glucosidase effects with an $\mathrm{IC}_{50}$ : $54.1 \mu \mathrm{M}$. On the other hand, compound 63 illustrated potent $\alpha$-glucosidase effects with $\mathrm{IC}_{50}: 8.1 \mu \mathrm{M}$ and its activity was higher than the standard acarbose $\mathrm{IC}_{50}$ : $703.8 \mu \mathrm{M}$ (Zhang et al. 2017). Moreover botryorhodines $C(64)$ and D (62) possess significant $\alpha$-glucosidase effects with $\mathrm{IC}_{50}$ : 11.2 and $10.3 \mu \mathrm{M}$ (Zhang et al. 2017) respectively and these metabolites were reported from the fungi Trichoderma sp. (Zhang et al. 2017) and Botryosphaeria rhodian (Abdou et al. 2010). All these compounds illustrated $\alpha$ glucosidase activity with $\mathrm{IC}_{50}$ values ranging from<smiles>[R]c1oc(=O)c2c([R])cc(O)c([R2])c2c1[R]</smiles>

8.1 to $54.1 \mu \mathrm{M}$ (Table 2). Compared to the reference drug acarbose $\left(\mathrm{IC}_{50}=703.8 \mu \mathrm{M}\right)$, it can be concluded that compounds 62-64 are potent anti-diabetic depsidones. An SAR study showed that C-3 groups could affect $\alpha$-glucosidase effects (compound 63 vs. 62 vs. 59) while on the other hand the C- $3^{\prime}$ methyl group exerts no effect on the activity (compound 64 vs. 62) (Zhang et al. 2017).

\section{Isocoumarins}

Isocoumarins are a class secondary metabolites bearing a lactone core and these compounds feature a wide range of chemical diversity with a most diverse range of biological effects. Notably, some isocoumarins have been entered into clinical trials for cancer and other diseases (Hampl et al. 2011; Yin et al. 2001; Salloum et al. 2000; Pochet et al. 2004). The fungus Talaromyces amestolkiae produced the library of isocoumarins (65-79) (Fig. 9) which were evaluated for their $\alpha$-glucosidase inhibition. Isocoumarins $\mathbf{6 6}$, 70, 72 and 74 illustrated the most encouraging effects among all the tested compounds with $\mathrm{IC}_{50}$ values ranging from 17.2 to $89.4 \mu \mathrm{M}$ (Table 2). Furthermore metabolites $\mathbf{6 5}, \mathbf{7 5}, \mathbf{7 8}$ and $\mathbf{7 9}$ are interestingly, five-

66: $\mathrm{R}_{1}=\mathrm{OH}, \mathrm{R}_{2}=\mathrm{OMe}, \mathrm{R}_{3}=\mathrm{H}, \mathrm{R}_{4}=\mathrm{Me}$

67: $R_{1}=O M e, R_{2}=H, R_{3}=R_{4}=M e$

68: $R_{1}=O M e, R_{2}=R_{4}=H, R_{3}=C H(O H) M e$

69: $\mathrm{R}_{1}=\mathrm{R}_{2}=\mathrm{OH}, \mathrm{R}_{3}=\mathrm{CH}(\mathrm{OH}) \mathrm{Me}, \mathrm{R}_{4}=\mathrm{H}$

70: $\mathrm{R}_{1}=\mathrm{OH}, \mathrm{R}_{2}=\mathrm{H}, \mathrm{R}_{3}=\mathrm{CH}_{2} \mathrm{OH}, \mathrm{R}_{4}=\mathrm{Me}$

71: $\mathrm{R}_{1}=\mathrm{OMe}, \mathrm{R}_{2}=\mathrm{H}, \mathrm{R}_{3}=\mathrm{CH}_{2} \mathrm{OH}, \mathrm{R}_{4}=\mathrm{Me}$

72: $\mathrm{R}_{1}=\mathrm{OH}, \mathrm{R}_{2}=\mathrm{H}, \mathrm{R}_{3}=\mathrm{R}_{4}=\mathrm{Me}$

73: $\mathrm{R}_{1}=\mathrm{OH}, \mathrm{R}_{2}=\mathrm{H}, \mathrm{R}_{3}=\mathrm{CH}(\mathrm{OH}) \mathrm{Me}, \mathrm{R}_{4}=\mathrm{H}$

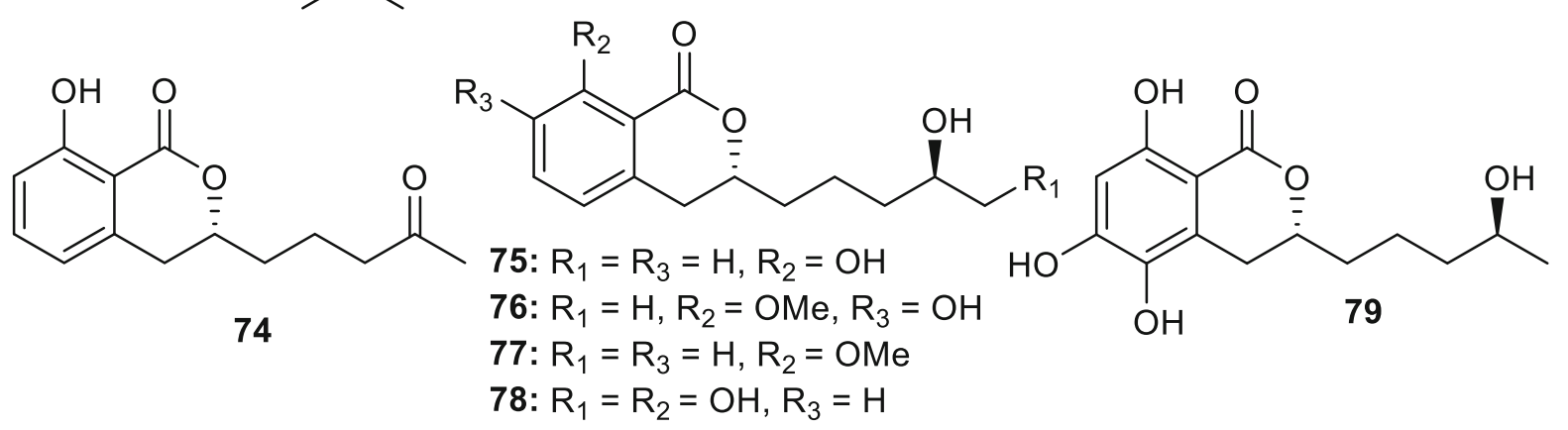

Fig. 9 Structures of isocoumarins 65-79 
fold more potent ( $\mathrm{IC}_{50}$ range: 140.8 to $193.1 \mu \mathrm{M}$ ) than acarbose $\left(\mathrm{IC}_{50}: 958.3 \mu \mathrm{M}\right)$ (Chen et al. 2016). Since metabolites 69, 71, 73, 76 and $\mathbf{7 7}$ illustrated moderate inhibition with $\mathrm{IC}_{50}$ ranging from 266.3 to $431.4 \mu \mathrm{M}$, respectively, it may be concluded that the 4- $\mathrm{CH}(\mathrm{OH}) \mathrm{CH}_{3}$ moiety in metabolites 68,69 and 73 decreases the activity level when compared with the activties with metabolites $\mathbf{7 0}$ and $\mathbf{7 1}$ bearing a 4-hydroxymethyl group. The compounds comprising an alkyl chain with the carbonyl group (as in compound 74) appear to possess higher activity in competition with compounds 75-79 which only have an hydroxyl moiety at the corresponding position (Chen et al. 2016).

The fungus Aspergillus sp. produces a range of furo[3,2- $h]$ isocoumarin derivatives viz., asperisocoumarins A $\left(\mathbf{8 0}: \mathrm{IC}_{50}: 87.8 \mu \mathrm{M}\right), \mathrm{E}\left(\mathbf{8 1}: \mathrm{IC}_{50}\right.$ : $52.3 \mu \mathrm{M})$, and $\mathrm{F}\left(\mathbf{8 2}: \mathrm{IC}_{50}: 95.6 \mu \mathrm{M}\right)$ and all displayed moderated $\alpha$-glucosidase effects (Table 3 ) (Xiao et al. 2016). Furo[3,2- $h]$ isocoumarins are uncommon and few members of this group have been reported in literature (Saeed 2016; Baba et al. 1991). Asperisocoumarin $\mathrm{E}(\mathbf{8 1})$ featured an isopentenyl group having two adjacent carbonyl moieties and asperisocoumarin F (82) represents an isocoumarin dimer via an ether linkage (Xiao et al. 2016). Furthermore, the same fungus Aspergillus sp. produced other isocoumarin analogues viz., asperisocoumarins $\mathrm{C}(\mathbf{8 3}), \mathrm{E}(\mathbf{8 4}), \mathrm{F}$ (85), G (86), I (87), J (89) (Fig. 10) along with compound $\mathbf{8 8}$ and their structures were all established via extensive NMR spectroscopic techniques. It was found that metabolites $83,86,87$, and 89 illustrated significant $\alpha$-glucosidase activities with $\mathrm{IC}_{50}$ ranging from 38.1 to $78.1 \mu \mathrm{M}$. Since metabolites $\mathbf{8 4}$ and $\mathbf{8 5}$ displayed only moderate inhibition (Table 3), it may be concluded that either an epoxide and/or an hydroxyl group at $\mathrm{C}$-10/C-11 could enhance the $\alpha$-glucosidase effects (Cai et al. 2018).

The isocoumarin 12-epicitreoisocoumarinol (90) is produced by the fungus Nectria sp., and illustrated $\alpha$ glucosidase potential with $\mathrm{IC}_{50}: 343.7 \mu \mathrm{M}$ (Cui et al. 2016). On the other hand citreoisocoumarinol (91) and citreoisocoumarin (92) (Fig. 11) were produced by the fungi Nectria sp. (Cui et al. 2016), Fusarium sp. (Ola et al. 2013) and Penicillium sp. (Lai et al. 1991) and display activity towards $\alpha$-glucosidase with $\mathrm{IC}_{50}$ : 392.5 and $538.7 \mu \mathrm{M}$ (Table 3) respectively (Cui et al. 2016). Notably, compounds 90-92 were more potent than acarbose $\left(\mathrm{IC}_{50}=815.3 \mu \mathrm{M}\right)$ (Cui et al.
2016). The fungus, Aspergillus sp. produced 6-Odemethylmonocerin (93: $\left.\mathrm{IC}_{50}: 0.027 \mathrm{mM}\right),(+)$ monocerin $\left(94 ; \mathrm{IC}_{50}: 1.65 \mathrm{mM}\right)$, fusarentin 6-methyl ether $\left(\mathbf{9 5} ; \mathrm{IC}_{50}: 1.19 \mathrm{mM}\right)$ and 6,7-O-dimethyl-4Rhydroxy-10-epifusarentin $\left(\mathbf{9 6} ; \mathrm{IC}_{50}: 1.74 \mathrm{mM}\right)$ and all were shown to possess $\alpha$-glucosidase effects (Table 3). Among these metabolites, compound $\mathbf{9 3}$ displayed 35 times more potent inhibitory effects than acarbose $\left(\mathrm{IC}_{50}=0.95 \mathrm{mM}\right)($ Kong et al. 2015).

\section{$\beta$-Resorcylic acid derivatives}

$\beta$-Resorcylic acid analogs have been reported from a number of natural sources and these compounds were reported to display a diverse range of biological activities viz., antimicrobial (Yang et al. 2006), antimalarial (Xu et al. 2010), cytotoxic (Buayairaksa et al. 2011) and kinases and ATPases inhibitions (Shen et al. 2015). In this regard, the fungus Lasiodiplodia sp. produced $\beta$-resorcylic acid analogs 97-99 (Fig. 12) and their structures were established by 1D, 2D NMR, and X-ray crystallography in addition to the Mosher protocol (Chen et al. 2015b). All the compounds were evaluated for their $\alpha$-glucosidase effects and illustrated inhibition with $\mathrm{IC}_{50}$ values ranging from 15.2 to $24.6 \mu \mathrm{M}$ (Table 3 ) and interestingly, their activities were better than the standard acarbose $\left(\mathrm{IC}_{50}=368 \mu \mathrm{M}\right)$ (Chen et al. 2015b). Moreover compound 100, which was reported from the fungus Lasiodiplodia sp. (Chen et al. 2015b) and the yeast Saccharomyces cerevisiae (Xu et al. 2014), proved to be a most potent $\alpha$-glucosidase inhibitor with an $\mathrm{IC}_{50}: 10.1 \mu \mathrm{M}$ (Chen et al. 2015b).

Compound 101, initially reported as a plant metabolite viz., from Euphorbia splendenfs (Lee et al. 1982) and later from the fungus Lasiodiplodia sp. (Chen et al. 2015b), illustrated $\alpha$-glucosidase effects with $\mathrm{IC}_{50}$ : $32.5 \mu \mathrm{M}$ (Chen et al. 2015b). Compound $\mathbf{1 0 2}$ was obtained from the fungus $\mathrm{La}$ siodiplodia sp. (Chen et al. 2015b; Aldridge et al. 1971), and compound $\mathbf{1 0 3}$ from the fungi Lasiodiplodia sp. (Chen et al. 2015b) as well as ZZF36 (Yang et al. 2006). Both compounds inhibited $\alpha$-glucosidase activity with $\mathrm{IC}_{50}$ : 13.6 and $35.9 \mu \mathrm{M}$ respectively (Chen et al. 2015b). SAR studies for compounds 97-103 demonstrated that the C-3 hydroxyl moiety in these compounds enhances the inhibitory effects (compound 99 vs. 100 and compound 101 vs. 102) 
Table 3 Fungal metabolites $\mathbf{7 4 - 1 1 2}$ as $\alpha$-glucosidase inhibitors

\begin{tabular}{|c|c|c|c|}
\hline Compd. & Source & $\begin{array}{l}\text { A-Glucosidase } \\
\text { activity }\end{array}$ & References \\
\hline 21,22 aspergillumarin A (74) & $\begin{array}{l}\text { Talaromyces } \\
\text { amestolkiae }\end{array}$ & $\mathrm{IC}_{50}: 38.1 \mu \mathrm{M}$ & $\begin{array}{l}\text { Chen et al. } \\
\text { (2016) }\end{array}$ \\
\hline 24 aspergillumarin B (75) & $\begin{array}{l}\text { Talaromyces } \\
\text { amestolkiae }\end{array}$ & $\mathrm{IC}_{50}: 193.1 \mu \mathrm{M}$ & $\begin{array}{l}\text { Chen et al. } \\
\text { (2016) }\end{array}$ \\
\hline 24penicimarin $\mathrm{C}$ (76) & $\begin{array}{l}\text { Talaromyces } \\
\text { amestolkiae }\end{array}$ & $\mathrm{IC}_{50}: 266.3 \mu \mathrm{M}$ & $\begin{array}{l}\text { Chen et al. } \\
\text { (2016) }\end{array}$ \\
\hline 26 and penicimarin $\mathrm{B}(\mathbf{7 7})$ & $\begin{array}{l}\text { Talaromyces } \\
\text { amestolkiae }\end{array}$ & $\mathrm{IC}_{50}: 431.4 \mu \mathrm{M}$ & $\begin{array}{l}\text { Chen et al. } \\
\text { (2016) }\end{array}$ \\
\hline Compound $\mathbf{7 8}$ & $\begin{array}{c}\text { Talaromyces } \\
\text { amestolkiae }\end{array}$ & $\mathrm{IC}_{50}: 162.5 \mu \mathrm{M}$ & $\begin{array}{c}\text { Chen et al. } \\
\text { (2016) }\end{array}$ \\
\hline Compound 79 & $\begin{array}{l}\text { Talaromyces } \\
\text { amestolkiae }\end{array}$ & $\mathrm{IC}_{50}: 142.1 \mu \mathrm{M}$ & $\begin{array}{c}\text { Chen et al. } \\
\text { (2016) }\end{array}$ \\
\hline Asperisocoumarin A $(\mathbf{8 0})$ & Aspergillus sp. & $\mathrm{IC}_{50}: 87.8 \mu \mathrm{M}$ & $\begin{array}{l}\text { Xiao et al. } \\
\text { (2016) }\end{array}$ \\
\hline Asperisocoumarin E $(\mathbf{8 1})$ & Aspergillus sp. & $\mathrm{IC}_{50}: 52.3 \mu \mathrm{M}$ & $\begin{array}{l}\text { Xiao et al. } \\
\text { (2016) }\end{array}$ \\
\hline Asperisocoumarin F (82) & Aspergillus sp. & $\mathrm{IC}_{50}: 95.6 \mu \mathrm{M}$ & $\begin{array}{l}\text { Xiao et al. } \\
\text { (2016) }\end{array}$ \\
\hline Asperisocoumarin C (83) & Aspergillus sp. & $\mathrm{IC}_{50}: 38.1 \mu \mathrm{M}$ & $\begin{array}{l}\text { Cai et al. } \\
\text { (2018) }\end{array}$ \\
\hline Asperisocoumarin E (84) & Aspergillus sp. & $\mathrm{IC}_{50}: 158.4 \mu \mathrm{M}$ & $\begin{array}{l}\text { Cai et al. } \\
\text { (2018) }\end{array}$ \\
\hline Asperisocoumarin F (85) & Aspergillus sp. & $\mathrm{IC}_{50}: 110.3 \mu \mathrm{M}$ & $\begin{array}{l}\text { Cai et al. } \\
\text { (2018) }\end{array}$ \\
\hline Asperisocoumarin G $(\mathbf{8 6})$ & Aspergillus sp. & $\mathrm{IC}_{50}: 40.5 \mu \mathrm{M}$ & $\begin{array}{c}\text { Cai et al. } \\
\text { (2018) }\end{array}$ \\
\hline Asperisocoumarin I (87) & Aspergillus sp. & $\mathrm{IC}_{50}: 78.1 \mu \mathrm{M}$ & $\begin{array}{l}\text { Cai et al. } \\
\text { (2018) }\end{array}$ \\
\hline $\begin{array}{l}\text { 3-[(R)-3,3-dichloro-2-hydroxypropyl]-8-hydroxy- } \\
\text { 6-methoxy-1H-isochromen-1-one }(\mathbf{8 8})\end{array}$ & Aspergillus sp. & $\mathrm{IC}_{50}: 102.4 \mu \mathrm{M}$ & $\begin{array}{l}\text { Cai et al. } \\
\text { (2018) }\end{array}$ \\
\hline Asperisocoumarin J (89) & Aspergillus sp. & $\mathrm{IC}_{50}: 45.1 \mu \mathrm{M}$ & $\begin{array}{l}\text { Cai et al. } \\
\text { (2018) }\end{array}$ \\
\hline 12-Epicitreoisocoumarinol (90) & Nectria sp. & $\mathrm{IC}_{50}: 343.7 \mu \mathrm{M}$ & $\begin{array}{l}\text { Cui et al. } \\
\text { (2016) }\end{array}$ \\
\hline Citreoisocoumarinol (91) & Nectria sp. & $\mathrm{IC}_{50}: 392.5 \mu \mathrm{M}$ & $\begin{array}{l}\text { Cui et al. } \\
\text { (2016) }\end{array}$ \\
\hline Citreoisocoumarin (92) & Nectria sp. & $\mathrm{IC}_{50}: 538.7 \mu \mathrm{M}$ & $\begin{array}{l}\text { Cui et al. } \\
\text { (2016) }\end{array}$ \\
\hline 6-O-demethylmonocerin (93) & Aspergillus sp. & $\mathrm{IC}_{50}: 0.027 \mathrm{mM}$ & $\begin{array}{l}\text { Kong et al. } \\
(2015)\end{array}$ \\
\hline$(+)$-monocerin $(\mathbf{9 4})$ & Aspergillus sp. & $\mathrm{IC}_{50}: 1.65 \mathrm{mM}$ & $\begin{array}{l}\text { Kong et al. } \\
\text { (2015) }\end{array}$ \\
\hline fusarentin 6-methyl ether (95) & Aspergillus sp. & $\mathrm{IC}_{50}: 1.19 \mathrm{mM}$ & $\begin{array}{l}\text { Kong et al. } \\
\text { (2015) }\end{array}$ \\
\hline 6,7-O-dimethyl-4R-hydroxy-10-epifusarentin (96) & Aspergillus sp. & $\mathrm{IC}_{50}: 1.74 \mathrm{mM}$ & $\begin{array}{l}\text { Kong et al. } \\
\text { (2015) }\end{array}$ \\
\hline (R)-ethyl 3,5-dihydroxy-7-(8-hydroxynonyl) benzoate (97) & Lasiodiplodia sp. & $\mathrm{IC}_{50}: 22.3 \mu \mathrm{M}$ & $\begin{array}{c}\text { Chen et al. } \\
\text { (2015b) }\end{array}$ \\
\hline
\end{tabular}


Table 3 continued

\begin{tabular}{|c|c|c|c|}
\hline Compd. & Source & $\begin{array}{l}\text { A-Glucosidase } \\
\text { activity }\end{array}$ & References \\
\hline$(R, E)$-ethyl 2,4-dihydroxy-6-(8-hydroxynon-1-en-1-yl) benzoate (98) & Lasiodiplodia sp. & $\mathrm{IC}_{50}: 24.6 \mu \mathrm{M}$ & $\begin{array}{l}\text { Chen } \\
\text { et al. (2015b) }\end{array}$ \\
\hline 3-Methoxy-lasicicol (99) & Lasiodiplodia $s p$. & $\mathrm{IC}_{50}: 15.2 \mu \mathrm{M}$ & $\begin{array}{l}\text { Chen } \\
\text { et al. (2015b) }\end{array}$ \\
\hline Lasicicol (100) & Lasiodiplodia $s p$. & $\mathrm{IC}_{50}: 10.1 \mu \mathrm{M}$ & $\begin{array}{l}\text { Chen et al. } \\
\text { (2015b) }\end{array}$ \\
\hline Lasiodiplodin (101) & Lasiodiplodia $s p$. & $\mathrm{IC}_{50}: 32.5 \mu \mathrm{M}$ & $\begin{array}{l}\text { Chen } \\
\text { et al. (2015b) }\end{array}$ \\
\hline De-O-methyllasiodiplodin (102) & Lasiodiplodia sp. & $\mathrm{IC}_{50}: 13.6 \mu \mathrm{M}$ & $\begin{array}{l}\text { Chen } \\
\text { et al. (2015b) }\end{array}$ \\
\hline (E)-9-etheno-lasiodiplodin (103) & Lasiodiplodia sp. & $\mathrm{IC}_{50}: 35.9 \mu \mathrm{M}$ & $\begin{array}{l}\text { Chen et al. } \\
(2015 b)\end{array}$ \\
\hline Lasiodiplactone A (104) & $\begin{array}{c}\text { Lasiodiplodia } \\
\text { theobromae }\end{array}$ & $\mathrm{IC}_{50}=367 \mu \mathrm{M}$ & $\begin{array}{l}\text { Chen et al. } \\
\text { (2017) }\end{array}$ \\
\hline Eurothiocin A (105) & Eurotium rubrum & $\mathrm{IC}_{50}=17.1 \mu \mathrm{M}$ & $\begin{array}{r}\text { Liu et al. } \\
(2014 a)\end{array}$ \\
\hline Eurothiocin B (106) & Eurotium rubrum & $\mathrm{IC}_{50}=42.6 \mu \mathrm{M}$ & $\begin{array}{r}\text { Liu et al. } \\
(2014 a)\end{array}$ \\
\hline Epicoccolide B (107) & Aspergillus flavipes & $\mathrm{IC}_{50}: 33 \mu \mathrm{M}$ & $\begin{array}{c}\text { Wang et al. } \\
(2016)\end{array}$ \\
\hline 6-Demethylpenisimplicissin (108) & Penicillium sp. & $\mathrm{IC}_{50}: 9.5 \mu \mathrm{M}$ & $\begin{array}{l}\text { Liu et al. } \\
\text { (2014b) }\end{array}$ \\
\hline $1^{\prime \prime}$-Epihydroxydihydrovermistatin (109) & Penicillium sp. & $\mathrm{IC}_{50}: 8.0 \mu \mathrm{M}$ & $\begin{array}{l}\text { Liu et al. } \\
\text { (2014b) }\end{array}$ \\
\hline Vermistatin (110) & Penicillium sp. & $\mathrm{IC}_{50}: 29.2 \mu \mathrm{M}$ & $\begin{array}{l}\text { Liu et al. } \\
\text { (2014b) }\end{array}$ \\
\hline Hydroxyvermistatin (111) & Penicillium sp. & $\mathrm{IC}_{50}: 20.3 \mu \mathrm{M}$ & $\begin{array}{l}\text { Liu et al. } \\
\text { (2014b) }\end{array}$ \\
\hline$(+)$-Geodin $(\mathbf{1 1 2})$ & & $\mathrm{IC}_{50}$ of $0.12 \mathrm{mM}$ & $\begin{array}{l}\text { Wang et al. } \\
\text { (2018) }\end{array}$ \\
\hline
\end{tabular}

(Table 3). Furthermore, when comparing the activity of compound 97 and 102, it may be assumed that the aliphatic macrocyclic acid system is also necessary for the activity (Chen et al. 2015b). Another 12-membered ring system comprising a $\beta$-resorcylic acid analog, lasiodiplactone A (104), reported from the fungus Lasiodiplodia theobromae comprises an interesting and unique 12/6/6/5 tetracyclic system. Compound 104 inhibited $\alpha$-glucosidase with an $\mathrm{IC}_{50}$ : $29.4 \mu \mathrm{M}$ which is better than the clinical acarbose $\left(\mathrm{IC}_{50}=367 \mu \mathrm{M}\right)($ Chen et al. 2017).

\section{Benzofurans}

The fungus Eurotium rubrum SH-823, produced the benzofuran analogs eurothiocin $\mathrm{A}\left(\mathbf{1 0 5} ; \mathrm{IC}_{50-}\right.$ $=17.1 \mu \mathrm{M})$ and $\mathrm{B}\left(\mathbf{1 0 6} ; \mathrm{IC}_{50}=42.6 \mu \mathrm{M}\right)($ Fig. 13), which inhibited the $\alpha$-glucosidase activity as illustrated in Table 4. It is worth mentioning that both compounds exhibited considerably lower $\mathrm{IC}_{50}$ values compared to the standard drug acarbose $\left(\mathrm{IC}_{50}\right.$ $=376.7 \mu \mathrm{M}$ ), which makes them interesting candidates for further studies (Liu et al. 2014a). In addition, epicoccolide B (107) was produce by the fungi Aspergillus flavipes (Wang et al. 2016) and Epicoccum sp. (Talontsi et al. 2013) and this compound inhibited $\alpha$-glucosidase with an $\mathrm{IC}_{50}$ of $33 \mu \mathrm{M}$ (Wang et al. 


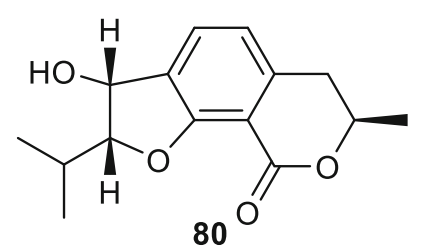<smiles>CC(C)C(=O)C(=O)c1ccc2c(c1O)C(=O)O[C@@H](C)C2</smiles><smiles>COc1cc2c(c(O)c1COCc1c(OC)cc3c(c1O)C(=O)OCC3)C(=O)OCC2</smiles><smiles>COc1cc(O)c2c(=O)oc(C[C@@H]3O[C@H]3C(C)=O)cc2c1</smiles><smiles>[R]c1c(OC)cc(O)c2c(=O)oc(C[C@H](O)C([R])[R])cc12</smiles><smiles>[R]c1c(OC)cc(O)c2c(=O)oc(C[C@H](Cl)CO)cc12</smiles><smiles>C[C@@H](O)Cc1cc2c(O)c(O)cc(O)c2c(=O)o1</smiles>

85: $\mathrm{R}=\mathrm{OH}$

87: $\mathrm{R}_{1}=\mathrm{R}_{2}=\mathrm{OH}, \mathrm{R}_{3}=\mathrm{H}$

88: $R_{1}=H, R_{2}=R_{3}=C l$

89: $\mathrm{R}_{1}=\mathrm{OH}, \mathrm{R}_{2}=\mathrm{R}_{3}=\mathrm{Cl}$

Fig. 10 Structures of isocoumarins 80-89

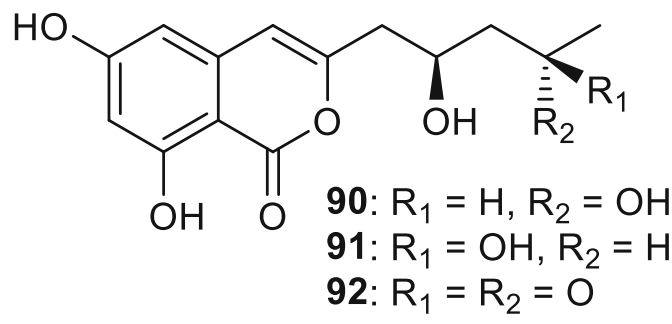<smiles>[R10]Oc1c(OC)cc2c(c1O)C(=O)O[C@H](CC(O)CCC)[C@@H]2[R2]</smiles>

93: $\mathrm{R}=\mathrm{H}$<smiles>[R6]Oc1cc2c(c(O)c1OC)C(=O)O[C@@H]1C[C@H](CCC)O[C@H]21</smiles>

94: $R=M e$

95: $R_{1}=R_{2}=H, 10 S$

96: $\mathrm{R}_{1}=\mathrm{Me}, \mathrm{R}_{2}=\mathrm{OH}, 10 \mathrm{R}$

Fig. 11 Structures of isocoumarins 90-96

2016). In addition, kinetic studies demonstrated that benzofuran 107 acted as a noncompetitive inhibitor with $\mathrm{K}_{\mathrm{i}}$ values of $2.5 / 7.2 \mu \mathrm{M}$, and thus have been identified as displaying mixed behavior (Wang et al. 2016).
Benzofuran analogs, 6-demethylpenisimplicissin (108), $1^{\prime \prime}$-epihydroxydihydrovermistatin (109), vermistatin (110) and hydroxyvermistatin (111) (Fig. 14) were obtained from Penicillium sp. and tested for their anti- $\alpha$-glucosidase effects. The results revealed that metabolites $108\left(\mathrm{IC}_{50}: 9.5 \mu \mathrm{M}\right)$ and $109\left(\mathrm{IC}_{50}\right.$ : $8.0 \mu \mathrm{M})$ illustrated significant activity while the $\alpha$ - 


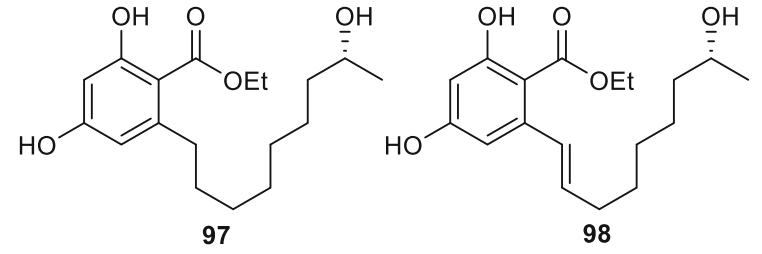

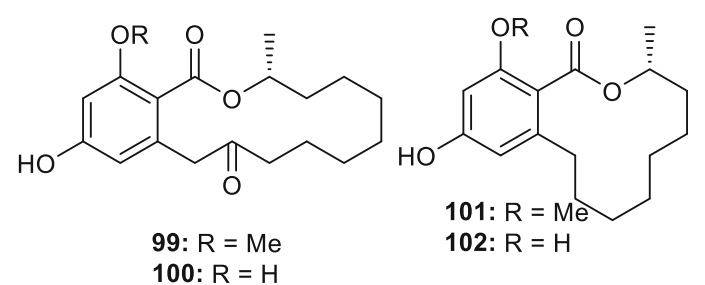

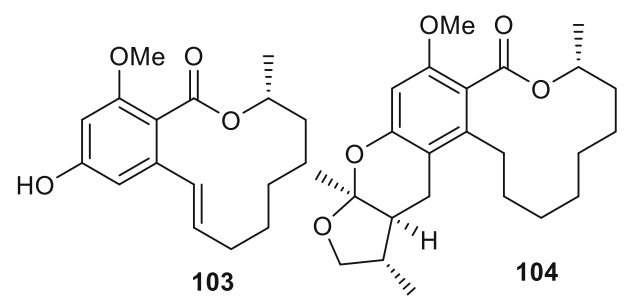

Fig. 12 Structures of $\beta$-resorcylic acid analogs 97-104

glucosidase inhibition capacity of compounds $\mathbf{1 1 0}$ $\left(\mathrm{IC}_{50}: 29.2 \mu \mathrm{M}\right)$ and $111\left(\mathrm{IC}_{50}: 20.3 \mu \mathrm{M}\right)$ (Table 4) were lower, but still higher that the reference resveratrol $\left(\mathrm{IC}_{50}=31.2 \mu \mathrm{M}\right)$ (Liu et al. 2014b). (+)-Geodin (112), which is a biosynthetically a polyketide derived compound (Askenazi et al. 2003; Couch and Gaucher 2004; Sutherland, et al. 2001), was reported from the fungus Penicillium chrysogenum (Wang et al. 2018) along with the fungus Aspergillus sp. (Hargreaves et al. 2002), illustrated excellent $\alpha$-glucosidase effects with an $\mathrm{IC}_{50}$ of $0.12 \mathrm{mM}$ (Wang et al. 2018).

\section{Quinones and xanthones}

Emodin (113) is reported from various plant families (Izhaki 2002) as well as various fungal species viz Penicillium sp. (Shibata and Udagawa 1963; Natori et al. 1965; Ghosh et al. 1977), Aspergillus sp. Yamazaki et al. 1971; Wells et al. 1975; Anke et al. 1980; Wang et al. 2016), Cladosporium fulvum (Agosti et al. 1962), and Penicilliopsis clavariaeformis (Shibata et al. 1957). Emodin (113) illustrated quite reasonable $\alpha$-glucosidase effects $\mathrm{IC}_{50}: 19 \mu \mathrm{M}$ and kinetic studies demonstrated that compound $\mathbf{1 1 3}$ (Fig. 15) was a noncompetitive inhibitor with a $\mathrm{K}_{\mathrm{i}}$ value of $0.79 \mu \mathrm{M}$ (Wang et al. 2016). On the other hand the fungus Alternaria sp. produced the tetrahydroanthraquinone analogs, alterporriol S (114), alterporriol $\mathrm{T}$ (115) and anthraquinone 116 (Chen et al. 2014). Quinone 115 ( $\left.\mathrm{IC}_{50}: 7.2 \mu \mathrm{M}\right)$ illustrated significant $\alpha$-glucosidase effects being higher than the positive control genistein $\left(\mathrm{IC}_{50}=13.6 \mu \mathrm{M}\right)$. In this regard it is interesting to note that metabolites $\mathbf{1 1 4}$ $\left(\mathrm{IC}_{50}=72.0 \mu \mathrm{M}\right)$ and $116\left(\mathrm{IC}_{50}=83.5 \mu \mathrm{M}\right)$ displayed weaker activity (Table 4) (Chen et al. 2014).

The xanthone derivatives (+)- and (-)-ascomlactone A (117a and 117b) (Fig. 16) are produced by the fungus Ascomycota sp. and their structures were established based on spectroscopic methods. The activity studies revealed that as a racemic mixture, these metabolites inhibited the activity of $\alpha$-glucosidase with an $\mathrm{IC}_{50}$ value of $36.1 \mu \mathrm{M}$. On the other hand, and interestingly, the pure enantiomer, (-)-ascomlactone A (117b) illustrated better inhibition with an $\mathrm{IC}_{50}$ value of $27.9 \mu \mathrm{M}$, whereas, the enantiomer (+)ascomlactone A (117a) demonstrated slightly weaker inhibition $\left(\mathrm{IC}_{50}=63.7 \mu \mathrm{M}\right)$. When compared to the activity of acarbose $\left(\mathrm{IC}_{50}=477.0 \mu \mathrm{M}\right)$, both pure enatiomers as well as the racemate is more potent (Liu et al. 2017). Chrysoxanthone (118) was obtained from the fungus Penicillium chrysogenum and illustrated $\alpha$ glucosidase effects with an $\mathrm{IC}_{50}$ of $0.04 \mathrm{mM}$ (Wang et al. 2018).

Fig. 13 Structures of benzofurans 105-107<smiles>[R]CC(C)(O)[C@H]1Cc2c(cc(C)c(C(=O)SC)c2O)O1</smiles> 
Table 4 Fungal metabolites $\mathbf{1 1 3 - 1 2 8}$ as $\alpha$-glucosidase inhibitors

\begin{tabular}{|c|c|c|c|}
\hline Compd. & Source & $\begin{array}{l}\text { A-Glucosidase } \\
\text { activity }\end{array}$ & References \\
\hline Emodin (113) & Aspergillus flavipes & $\begin{array}{l}\mathrm{IC}_{50}: 19 \mu \mathrm{M} ; \mathrm{K}_{\mathrm{i}} \text { : } \\
0.79 \mu \mathrm{M}\end{array}$ & $\begin{array}{l}\text { Wang et al. } \\
\text { (2016) }\end{array}$ \\
\hline Alterporriol S (114) & Alternaria sp. & $\mathrm{IC}_{50}=72.0 \mu \mathrm{M}$ & $\begin{array}{l}\text { Chen et al. } \\
\text { (2014) }\end{array}$ \\
\hline Alterporriol T (115) & Alternaria sp. & $\mathrm{IC}_{50}: 7.2 \mu \mathrm{M}$ & $\begin{array}{l}\text { Chen et al. } \\
\text { (2014) }\end{array}$ \\
\hline Macrosporin (116) & Alternaria sp. & $\mathrm{IC}_{50}=83.5 \mu \mathrm{M}$ & $\begin{array}{l}\text { Chen et al. } \\
\text { (2014) }\end{array}$ \\
\hline (+)-Ascomlactone A (117a) & Ascomycota sp. & $\mathrm{IC}_{50}=63.7 \mu \mathrm{M}$ & $\begin{array}{l}\text { Liu et al. } \\
\text { (2017) }\end{array}$ \\
\hline (-)-Ascomlactone A (117b) & Ascomycota sp. & $\mathrm{IC}_{50}=27.9 \mu \mathrm{M}$ & $\begin{array}{l}\text { Liu et al. } \\
\text { (2017) }\end{array}$ \\
\hline Chrysoxanthone (118) & $\begin{array}{l}\text { Penicillium } \\
\text { chrysogenum }\end{array}$ & $\mathrm{IC}_{50}=0.04 \mathrm{mM}$ & $\begin{array}{l}\text { Wang et al. } \\
\text { (2018) }\end{array}$ \\
\hline Daldinione B (119) & $\begin{array}{l}\text { Daldinia } \\
\text { eschscholtzii }\end{array}$ & $\mathrm{IC}_{50}=38 \mu \mathrm{M}$ & $\begin{array}{l}\text { Liao et al. } \\
\text { (2019) }\end{array}$ \\
\hline Daldinione C (120) & $\begin{array}{l}\text { Daldinia } \\
\text { eschscholtzii }\end{array}$ & $\mathrm{IC}_{50}=155 \mu \mathrm{M}$ & $\begin{array}{l}\text { Liao et al. } \\
\text { (2019) }\end{array}$ \\
\hline Daldinione D (121) & $\begin{array}{l}\text { Daldinia } \\
\text { eschscholtzii }\end{array}$ & $\mathrm{IC}_{50}=35 \mu \mathrm{M}$ & $\begin{array}{l}\text { Liao et al. } \\
\text { (2019) }\end{array}$ \\
\hline 4R-(O)-Methylsclerone (122) & $\begin{array}{l}\text { Daldinia } \\
\text { eschscholtzii }\end{array}$ & $\mathrm{IC}_{50}=90 \mu \mathrm{M}$ & $\begin{array}{l}\text { Liao et al. } \\
\text { (2019) }\end{array}$ \\
\hline Isosclerone (123) & $\begin{array}{l}\text { Daldinia } \\
\text { eschscholtzii }\end{array}$ & $\mathrm{IC}_{50}=70 \mu \mathrm{M}$ & $\begin{array}{l}\text { Liao et al. } \\
\text { (2019) }\end{array}$ \\
\hline $\begin{array}{l}(-) \text {-cis- }\left(3 R^{*}, 4 S^{*}\right)-3,4,8 \text {-trihydroxy-6,7-dimethyl-3,4- } \\
\text { dihydronaphthalen-1(2H)-one }(\mathbf{1 2 4})\end{array}$ & $\begin{array}{l}\text { Daldinia } \\
\text { eschscholtzii }\end{array}$ & $\mathrm{IC}_{50}=21 \mu \mathrm{M}$ & $\begin{array}{l}\text { Liao et al. } \\
\text { (2019) }\end{array}$ \\
\hline 7-Hydroxy-5-methoxy-2,3-dimethylchromone (125) & $\begin{array}{l}\text { Daldinia } \\
\text { eschscholtzii }\end{array}$ & $\mathrm{IC}_{50}=13 \mu \mathrm{M}$ & $\begin{array}{l}\text { Liao et al. } \\
\quad(2019)\end{array}$ \\
\hline 5-Methoxy-2-propylchromone (126) & $\begin{array}{l}\text { Daldinia } \\
\text { eschscholtzii }\end{array}$ & $\mathrm{IC}_{50}=84 \mu \mathrm{M}$ & $\begin{array}{l}\text { Liao et al. } \\
\quad(2019)\end{array}$ \\
\hline 7-Ethyl-8-hydroxy-6-methoxy-2,3-dimethylchromone (127) & $\begin{array}{l}\text { Daldinia } \\
\text { eschscholtzii }\end{array}$ & $\mathrm{IC}_{50}=100 \mu \mathrm{M}$ & $\begin{array}{l}\text { Liao et al. } \\
\text { (2019) }\end{array}$ \\
\hline 2,3-Dihydro-5-methoxy-2-methylchromen-4-one (128) & $\begin{array}{l}\text { Daldinia } \\
\text { eschscholtzii }\end{array}$ & $\mathrm{IC}_{50}=15 \mu \mathrm{M}$ & $\begin{array}{l}\text { Liao et al. } \\
\text { (2019) }\end{array}$ \\
\hline
\end{tabular}

\section{Tetralone and chromone derivatives}

The fungus Daldinia eschscholtzii produced a small library of tetralones viz., daldiniones B-D (119-121) and tetralones 122-124 (Fig. 17) and their structures were established by NMR, GIAO based NMR and ECD spectroscopic methods. All these compounds illustrated $\alpha$-glucosidase effects with $\mathrm{IC}_{50}$ values ranging from 21 to $155 \mu \mathrm{M}$ (Table 5). Among these compounds, metabolite $\mathbf{1 2 4}$ was the most active $\left(\mathrm{IC}_{50}\right.$ : $21 \mu \mathrm{M})$ followed by compounds $121\left(\mathrm{IC}_{50}: 35 \mu \mathrm{M}\right)$ and $119\left(\mathrm{IC}_{50}: 38 \mu \mathrm{M}\right)$ (Liao et al. 2019). Moreover, Daldinia eschscholtzii also produced the chromone analogs, 125-128, which were also tested for their $\alpha$ glucosidase effects. All these compounds inhibited $\alpha$ glucosidase with $\mathrm{IC}_{50}$ : ranging from 13 to $100 \mu \mathrm{M}$ (Table 5). Notably, chromone analogs $125\left(\mathrm{IC}_{50}\right.$ : $13 \mu \mathrm{M})$ and $128\left(\mathrm{IC}_{50}: 15 \mu \mathrm{M}\right)$ proved to be the most active metabolites among the tested chromones (Liao et al. 2019). 
<smiles>[R]c1cc(OC)c2c(c1)C(=O)O[C@H]2c1coc([R3])c([R])c1=O</smiles>

108: $R_{1}=O H, R_{2}=H, R_{3}=M e$<smiles>COC(=O)C1=C(O)C(=O)C=C(C)[C@]12Oc1c(Cl)c(C)c(Cl)c(O)c1C2=O</smiles>

112

Fig. 14 Structures of benzofurans 108-112

Fig. 15 Structures of quinones 113-116<smiles>[R]Oc1cc(O)c2c(c1)C(=O)c1cc([R3])c([R2])c(O)c1C2=O</smiles>

113: $R_{1}=R_{2}=H, R_{3}=M e$

116: $R_{1}=R_{2}=M e, R_{3}=H$<smiles>COc1cc(C)c2c(c1-c1c(OC)cc(O)c3c1C(=O)c1cc(C)c(O)cc1C3=O)C(=O)C1=C(C2=O)[C@@H](O)[C@@H](O)[C@](C)(O)[C@H]1O</smiles>

115<smiles>C=C[C@H]1C2=C(C[C@@](C)(O)[C@H]1O)C(=O)c1cc(OC)cc(O)c1C2=O</smiles>

\section{Butenolides}

The fungus Aspergillus terreus produces ( \pm -asperteretals D (129) and E (130), flavipesolides B (131) and C (132), butyrolactones I (133) and II (134), butanolide 135 and aspernolide A (136) (Fig. 18). All these metabolites illustrated $\alpha$-glucosidase inhibition with $\mathrm{IC}_{50}$ values ranging from 7.6 to $85.1 \mu \mathrm{M}$ (Table 5). Moreover, compound $\mathbf{1 3 2}$ proved to be the most potent since it displayed the lowest $\mathrm{IC}_{50}$ value of $7.63 \mu \mathrm{M}$. Both enantiomerically pure isomers of compound $\mathbf{1 2 9}$ also exerted significant inhibition of the enzyme with $\mathrm{IC}_{50}$ values of $8.65 \mu \mathrm{M}[(+)-129)$ and $9.98 \mu \mathrm{M}[(-)$ 129] (Sun et al. 2018a). It has been reported that $(+)$ and (-)-129 are methanolysis artifacts of asperteretal E (Capon 2020; Sun et al. 2018b).

The fungus $A$. terreus furthermore produced $( \pm)$ asperteretones A-D (137a/b-140a/b) (Fig. 19), and 

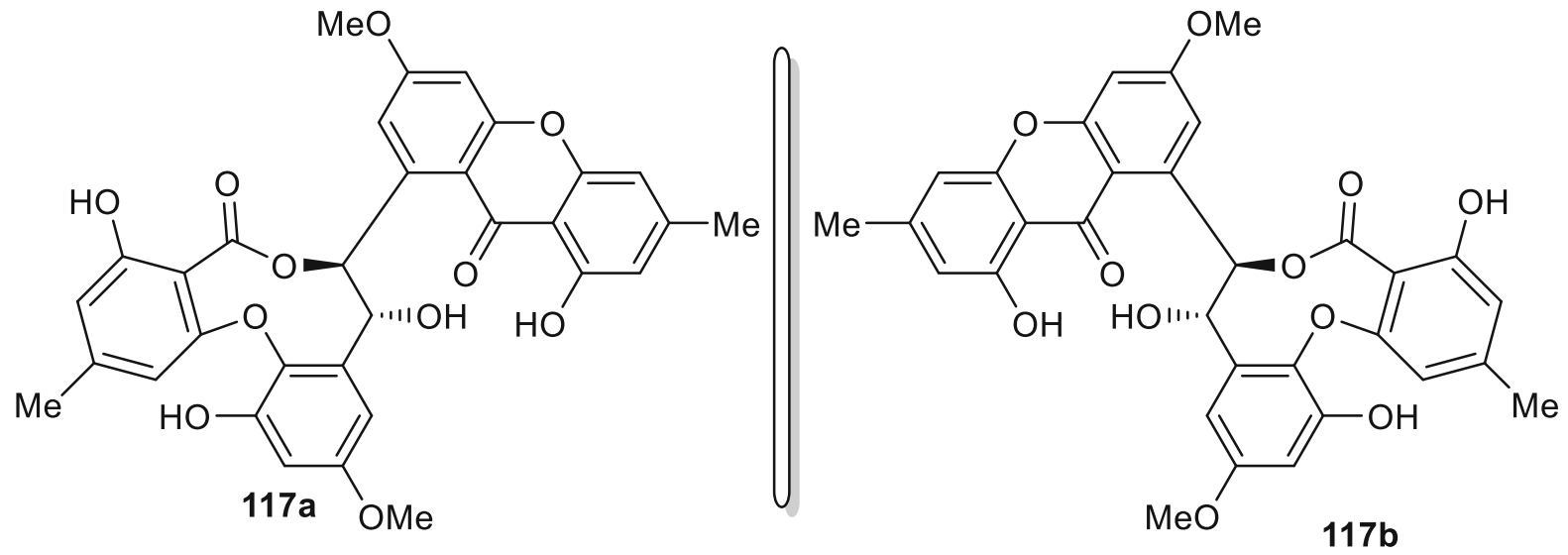<smiles>COC(=O)c1c(O)c(O)cc2oc3c(Cl)c(C)c(Cl)c(O)c3c(=O)c12</smiles>

Fig. 16 Structures of quinones xanthones $117 \mathbf{a}, \mathbf{b}$ and 118<smiles>CCc1ccc2c(c1O)C(=O)[C@@](O)(CC)[C@H](O)C2</smiles>

$$
\begin{aligned}
& \text { 120: } R=\beta-O H \\
& \text { 121: } R=\alpha-O H
\end{aligned}
$$<smiles>COc1cc(O)cc2oc(C)c(C)c(=O)c12</smiles>

125<smiles>CCCc1cc(=O)c2c(OC)cccc2o1</smiles>

126<smiles>[R2]c1ccc([R2])c2c1C(=O)CC[C@@H]2O</smiles><smiles>Cc1cc2c(c(O)c1C)C(=O)C[C@@H](O)[C@H]2O</smiles>

122: $R_{1}=O M e, R_{2}=H$

123: $R_{1}=H, R_{2}=O H$<smiles>CCc1c(OC)cc2c(=O)c(C)c(C)oc2c1O</smiles>

127<smiles>COc1cccc2c1C(=O)C[C@@H](C)O2</smiles>

128

Fig. 17 Structures of tetralone and chromone derivatives 119-128

the racemate, asperteretone $\mathrm{E}(\mathbf{1 4 1})$ and all these metabolites demonstrated $\alpha$-glucosidase inhibition with $\mathrm{IC}_{50}$ values ranging from 15.7 to $53.1 \mu \mathrm{M}$ (Table 6). Butanolide 140a $\left(\mathrm{IC}_{50}: 15.7 \mu \mathrm{M}\right)$ was the most active while $138 \mathrm{a}\left(\mathrm{IC}_{50}: 17.3 \mu \mathrm{M}\right), \mathbf{1 3 8 b}\left(\mathrm{IC}_{50}\right.$ : $19.2 \mu \mathrm{M})$ and $\mathbf{1 4 0 b}\left(\mathrm{IC}_{50}: 18.9 \mu \mathrm{M}\right)$ also displayed comparable inhibitory potential. Although the other compounds showed moderate activity, their inhibition values were higher than the reference drug acarbose $\left(\mathrm{IC}_{50}=154.7 \mu \mathrm{M}\right)($ Liu et al. 2018a). Notably, it was postulated that all enantiomers illustrated would most likely demonstrate similar $\alpha$-glucosidase effects, suggesting that chirality may play a negligible role on their potential activities. 
Table 5 Fungal metabolites $\mathbf{1 2 9 - 1 5 2}$ as $\alpha$-glucosidase inhibitors

\begin{tabular}{|c|c|c|c|}
\hline Compd. & Source & $\begin{array}{l}\text { A-Glucosidase } \\
\text { activity }\end{array}$ & References \\
\hline (-)-Asperteretal D (129) & $\begin{array}{c}\text { Aspergillus } \\
\text { terreus }\end{array}$ & $\mathrm{IC}_{50}=9.9 \mu \mathrm{M}$ & $\begin{array}{l}\text { Sun et al. } \\
(2018 a)\end{array}$ \\
\hline (+)-Asperteretal D (129) & $\begin{array}{l}\text { Aspergillus } \\
\text { terreus }\end{array}$ & $\mathrm{IC}_{50}=8.6 \mu \mathrm{M}$ & $\begin{array}{r}\text { Sun et al. } \\
(2018 \mathrm{a})\end{array}$ \\
\hline Asperteretal E (130) & $\begin{array}{l}\text { Aspergillus } \\
\text { terreus }\end{array}$ & $\mathrm{IC}_{50}=13.3 \mu \mathrm{M}$ & $\begin{array}{r}\text { Sun et al. } \\
(2018 \mathrm{a})\end{array}$ \\
\hline Flavipesolide B (131) & $\begin{array}{l}\text { Aspergillus } \\
\text { terreus }\end{array}$ & $\mathrm{IC}_{50}=10.3 \mu \mathrm{M}$ & $\begin{array}{r}\text { Sun et al. } \\
(2018 \mathrm{a})\end{array}$ \\
\hline Flavipesolide C (132) & $\begin{array}{l}\text { Aspergillus } \\
\text { terreus }\end{array}$ & $\mathrm{IC}_{50}=7.63 \mu \mathrm{M}$ & $\begin{array}{l}\text { Sun et al. } \\
(2018 \mathrm{a})\end{array}$ \\
\hline Butyrolactone I (133) & $\begin{array}{l}\text { Aspergillus } \\
\text { terreus }\end{array}$ & $\mathrm{IC}_{50}=14.1 \mu \mathrm{M}$ & $\begin{array}{r}\text { Sun et al. } \\
(2018 \mathrm{a})\end{array}$ \\
\hline Butyrolactone II (134) & $\begin{array}{l}\text { Aspergillus } \\
\text { terreus }\end{array}$ & $\mathrm{IC}_{50}=85.3 \mu \mathrm{M}$ & $\begin{array}{l}\text { Sun et al. } \\
(2018 \mathrm{a})\end{array}$ \\
\hline $\begin{array}{l}\text { 5-[(3,4-dihydro-2,2-dimethyl-2H-1-benzopyran-6-yl)-methyl]-3-hydroxy-4-(4- } \\
\text { hydroxyphenyl)-2(5H)-furanone (135) }\end{array}$ & $\begin{array}{l}\text { Aspergillus } \\
\text { terreus }\end{array}$ & $\mathrm{IC}_{50}=11.6 \mu \mathrm{M}$ & $\begin{array}{r}\text { Sun et al. } \\
(2018 \mathrm{a})\end{array}$ \\
\hline Aspernolide A (136) & $\begin{array}{l}\text { Aspergillus } \\
\text { terreus }\end{array}$ & $\mathrm{IC}_{50}=47.3 \mu \mathrm{M}$ & $\begin{array}{r}\text { Sun et al. } \\
(2018 \mathrm{a})\end{array}$ \\
\hline (-)-Asperteretones A (137a) & $\begin{array}{c}\text { Aspergillus } \\
\text { terreus }\end{array}$ & $\mathrm{IC}_{50}=45.4 \mu \mathrm{M}$ & $\begin{array}{r}\text { Liu et al. } \\
\text { (2018a) }\end{array}$ \\
\hline (+)-Asperteretones A (137b) & $\begin{array}{c}\text { Aspergillus } \\
\text { terreus }\end{array}$ & $\mathrm{IC}_{50}=53.1 \mu \mathrm{M}$ & $\begin{array}{r}\text { Liu et al. } \\
\text { (2018a) }\end{array}$ \\
\hline (-)-Asperteretones B (138a) & $\begin{array}{c}\text { Aspergillus } \\
\text { terreus }\end{array}$ & $\mathrm{IC}_{50}=17.3 \mu \mathrm{M}$ & $\begin{array}{r}\text { Liu et al. } \\
\text { (2018a) }\end{array}$ \\
\hline$(+)$-Asperteretones B (138b) & $\begin{array}{c}\text { Aspergillus } \\
\text { terreus }\end{array}$ & $\mathrm{IC}_{50}=19.2 \mu \mathrm{M}$ & $\begin{array}{l}\text { Liu et al. } \\
\text { (2018a) }\end{array}$ \\
\hline (-)-Asperteretones C (139a) & $\begin{array}{c}\text { Aspergillus } \\
\text { terreus }\end{array}$ & $\mathrm{IC}_{50}=52.2 \mu \mathrm{M}$ & $\begin{array}{l}\text { Liu et al. } \\
\text { (2018a) }\end{array}$ \\
\hline (+)-Asperteretones C (139b) & $\begin{array}{c}\text { Aspergillus } \\
\text { terreus }\end{array}$ & $\mathrm{IC}_{50}=49.8 \mu \mathrm{M}$ & $\begin{array}{l}\text { Liu et al. } \\
\text { (2018a) }\end{array}$ \\
\hline (-)-Asperteretones C (140a) & $\begin{array}{c}\text { Aspergillus } \\
\text { terreus }\end{array}$ & $\mathrm{IC}_{50}=15.7 \mu \mathrm{M}$ & $\begin{array}{r}\text { Liu et al. } \\
\text { (2018a) }\end{array}$ \\
\hline$(+)$-Asperteretones C (140b) & $\begin{array}{c}\text { Aspergillus } \\
\text { terreus }\end{array}$ & $\mathrm{IC}_{50}=18.9 \mu \mathrm{M}$ & $\begin{array}{r}\text { Liu et al. } \\
(2018 a)\end{array}$ \\
\hline Asperteretone E (141) & $\begin{array}{c}\text { Aspergillus } \\
\text { terreus }\end{array}$ & $\mathrm{IC}_{50}=48.9 \mu \mathrm{M}$ & $\begin{array}{l}\text { Liu et al. } \\
\text { (2018a) }\end{array}$ \\
\hline $\begin{array}{l}\text { (R,E)-3-(2,2-Dimethylchroman-6-yl)-4-hydroxy-5-((2-(2-hydroxypropan-2-yl)-2,3- } \\
\text { dihydrobenzofuran-5-yl)methylene) furan-2(5H)-one (142) }\end{array}$ & $\begin{array}{l}\text { Aspergillus } \\
\text { terreus }\end{array}$ & $\mathrm{IC}_{50}=24.8 \mu \mathrm{M}$ & $\begin{array}{r}\text { Sun et al. } \\
(2018 b)\end{array}$ \\
\hline Rubrolide S (143) & $\begin{array}{l}\text { Aspergillus } \\
\text { terreus }\end{array}$ & $\begin{array}{l}\mathrm{IC}_{50}=1.2 \mu \mathrm{M} \\
\text { Ki: } 1.42 \mu \mathrm{M}\end{array}$ & $\begin{array}{r}\text { Sun et al. } \\
(2018 b)\end{array}$ \\
\hline Avipesolide A (144); & $\begin{array}{l}\text { Aspergillus } \\
\text { flavipes }\end{array}$ & $\begin{array}{l}\mathrm{IC}_{50}: 44 \mu \mathrm{M} ; K_{\mathrm{i}}: \\
\quad 2.4 \mu \mathrm{M}\end{array}$ & $\begin{array}{l}\text { Wang et al. } \\
\text { (2016) }\end{array}$ \\
\hline Avipesolide B (145); & $\begin{array}{l}\text { Aspergillus } \\
\text { flavipes }\end{array}$ & $\begin{array}{l}\mathrm{IC}_{50}: 57 \mu \mathrm{M} ; K_{\mathrm{i}}: \\
\quad 3.4 \mu \mathrm{M}\end{array}$ & $\begin{array}{l}\text { Wang et al. } \\
\text { (2016) }\end{array}$ \\
\hline Avipesolide C (146); & $\begin{array}{l}\text { Aspergillus } \\
\text { flavipes }\end{array}$ & $\begin{array}{l}\mathrm{IC}_{50}: 95 \mu \mathrm{M} ; K_{\mathrm{i}}: \\
\quad 9.2 \mu \mathrm{M}\end{array}$ & $\begin{array}{l}\text { Wang et al. } \\
\text { (2016) }\end{array}$ \\
\hline $\begin{array}{l}\text { 5-[(3,4-dihydro-2,2-dimethyl-2H-1-benzopyran-6-yl)methyl]-3-hydroxy-4-(4- } \\
\text { hydroxyphenyl)-2(5H)furanone (147) }\end{array}$ & $\begin{array}{l}\text { Aspergillus } \\
\text { flavipes }\end{array}$ & $\begin{array}{l}\mathrm{IC}_{50}: 34 \mu \mathrm{M} ; K_{\mathrm{i}}: \\
0.43 \mu \mathrm{M}\end{array}$ & $\begin{array}{c}\text { Wang et al. } \\
\text { (2016) }\end{array}$ \\
\hline
\end{tabular}


Table 5 continued

\begin{tabular}{|c|c|c|c|}
\hline Compd. & Source & $\begin{array}{l}\text { A-Glucosidase } \\
\text { activity }\end{array}$ & References \\
\hline Pulvinone P (148) & $\begin{array}{c}\text { Aspergillus } \\
\text { flavipes }\end{array}$ & $\mathrm{IC}_{50}: 0.079 \mathrm{mM}$ & $\begin{array}{c}\text { Zhang et al. } \\
\quad(2016 a)\end{array}$ \\
\hline Pulvinone Q (149) & $\begin{array}{l}\text { Aspergillus } \\
\text { flavipes }\end{array}$ & $\mathrm{IC}_{50}: 0.022 \mathrm{mM}$ & $\begin{array}{c}\text { Zhang et al. } \\
\text { (2016a) }\end{array}$ \\
\hline Methybutyrolactone III (150): & $\begin{array}{c}\text { Aspergillus } \\
\text { flavipes }\end{array}$ & $\mathrm{IC}_{50}: 0.016 \mathrm{mM}$ & $\begin{array}{c}\text { Zhang et al. } \\
\text { (2016a) }\end{array}$ \\
\hline Flavipesin B (151): & $\begin{array}{c}\text { Aspergillus } \\
\text { flavipes }\end{array}$ & $\mathrm{IC}_{50}: 0.385 \mathrm{mM}$ & $\begin{array}{l}\text { Zhang et al. } \\
\text { (2016a) }\end{array}$ \\
\hline Versicolactone G (152) & $\begin{array}{c}\text { Aspergillus } \\
\text { terreus }\end{array}$ & $\mathrm{IC}_{50}=154.7 \mu$ & $\begin{array}{l}\text { Liu et al. } \\
\text { (2018b) }\end{array}$ \\
\hline
\end{tabular}<smiles>CO[C@H]1OC(=O)C(Cc2ccc(O)c(CC=C(C)C)c2)=C1c1ccc(O)cc1CC1=C(c2ccc(O)cc2)[C@H](OC)OC1=O</smiles>

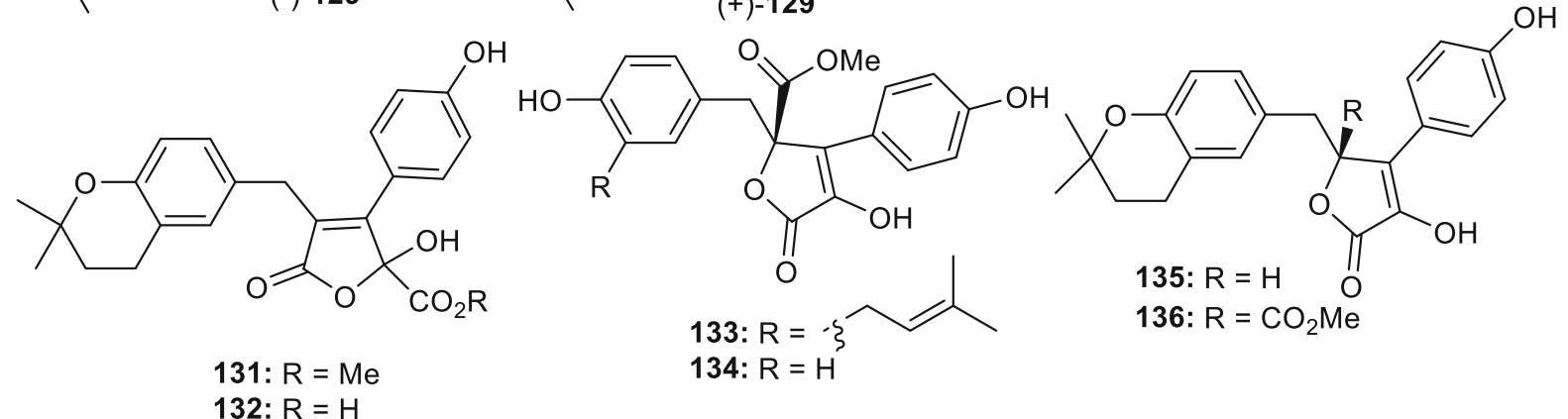

Fig. 18 Structures of butanolides 129-136

Further butenolides, compound $\mathbf{1 4 2}$ and rubrolide $\mathrm{S}$ (143) (Fig. 20) were obtained from the fungus $A$. terreus and demonstrated $\alpha$-glucosidase effects with $\mathrm{IC}_{50}$ values of 24.8 and $1.2 \mu \mathrm{M}$, respectively. Additional enzyme kinetic studies were performed with compound 143, which revealed that it is an anticompetitive inhibitor with a $K$ i value of $1.42 \mu \mathrm{M}$ (Sun et al. 2018b). Another fungus varietal of Aspergillus sp., viz., Aspergillus flavipes produced antidiabetic avipesolides A-C (144-146) and butanolide 147 and these compounds inhibit $\alpha$-glucosidase with $\mathrm{IC}_{50}$ values ranging from 34 to $95 \mu \mathrm{M}$ (Table 6).
Appropriate enzyme kinetic studies have also been carried out which revealed that compound $147\left(K_{\mathrm{i}}\right.$ : $0.43 \mu \mathrm{M})$ is a noncompetitive inhibitor while compounds 144-146 were demonstrated to be more competitive $\left(K_{\mathrm{i}}: 2.5,3.4\right.$, and $9.2 \mu \mathrm{M}$ respectively) (Wang et al. 2016).

Pulvinones have been reported from various fungi and illustrated interesting biological effects (Bernier et al. 2007; Xu et al. 2013). Pulvinones viz., pulvinone P (148: $\left.\mathrm{IC}_{50}: 0.079 \mathrm{mM}\right)$ and $\mathrm{Q}$ (149: $\mathrm{IC}_{50}$ : $0.022 \mathrm{mM}$ ) were reported from A. flavipes and illustrated significant $\alpha$-glucosidase inhibition (Zhang 
<smiles>COC(=O)[C@H](Cc1ccc(O)c(CC=C(C)C)c1)[C@H](C(=O)O)c1ccc(O)cc1</smiles><smiles>CO[C@H]1OC(=O)C(c2ccc(O)cc2)=C1Cc1ccc(O)c(CC=C(C)C)c1</smiles><smiles>COc1ccc(C2=C(Cc3ccc4c(c3)CC(C(C)(C)O)O4)[C@H](OC)OC2=O)cc1</smiles><smiles>CO[C@@H]1OC(=O)C(c2ccc(O)cc2)=C1Cc1ccc2c(c1)CCC(C)(C)O2</smiles>

$140 \mathrm{a}$<smiles>COC1OC(=O)C(c2ccc(O)cc2)=C1Cc1ccc(O)c(CCC(C)(C)O)c1</smiles><smiles>COC(=O)[C@H](Cc1ccc(O)c(CC=C(C)C)c1)[C@@H](C(=O)[18OH])c1ccc(O)cc1</smiles><smiles>CCCCC(C)(C)C</smiles><smiles>COc1ccc(C2=C(Cc3ccc4c(c3)CC(C(C)(C)O)O4)[C@H](OC)OC2=O)cc1</smiles><smiles>CO[C@H]1OC(=O)C(c2ccc(O)cc2)=C1Cc1ccc2c(c1)CCC(C)(C)O2</smiles>

$140 b$

Fig. 19 Structures of butanolides 137-141

et al. 2016a). Methybutyrolactone III (150: IC $_{50}$ : $0.016 \mathrm{mM})$ and flavipesin $\mathrm{B}\left(\mathbf{1 5 1}: \mathrm{IC}_{50}: 0.385 \mathrm{mM}\right)$ (Fig. 21) were obtained from A. flavipes and all were shown to possess significant $\alpha$-glucosidase inhibition (Table 6) when compared to the standard drug acarbose $\left(\mathrm{IC}_{50}=0.685 \mathrm{mM}\right.$ ) (Zhang et al. 2016a). A butenolide analog, versicolactone G (152) was obtained from the fungus Aspergillus terreus and showed inhibitory potential towards $\alpha$-glucosidase $\left(\mathrm{IC}_{50}=104.8 \mu \mathrm{M}\right)$ and under the same conditions this compound was more potent than the standard acarbose $\left(\mathrm{IC}_{50}=154.7 \mu \mathrm{M}\right)($ Liu et al. 2018b).

\section{Diphenyl ether and benzophenone derivatives}

Neogeodin hydrate (153) (Zhang et al. 2016a; Jongrungruangchok et al. 2013) and methyl dichloroasterrate (154) (Liu et al. 2015b ; Zhang et al. 2016a) were produced by various Aspergillus sp. and illustrated $\alpha$-glucosidase effects with $\mathrm{IC}_{50}: 1.47 \mathrm{mM}$ and $1.45 \mathrm{mM}$ respectively (Zhang et al. 2016a). 2,4Dichloroasterric acid (155) along with benzophenones 3-de-O-methylsulochrin (156) and compound 157 (Fig. 22) were also produced by Aspergillus sp. (Zhang et al. 2016a). All these compounds possess good $\alpha$-glucosidase inhibition with $\mathrm{IC}_{50}$ values 
Table 6 Fungal metabolites $\mathbf{1 5 3 - 1 8 9}$ as $\alpha$-glucosidase inhibitors

\begin{tabular}{|c|c|c|c|}
\hline Compd. & Source & $\begin{array}{l}\alpha \text {-Glucosidase } \\
\text { activity }\end{array}$ & References \\
\hline \multirow[t]{2}{*}{ Neogeodin hydrate (153), } & Aspergillus flavipes & $\mathrm{IC}_{50}: 1.47 \mathrm{mM}$ & $\begin{array}{l}\text { Zhang et al. } \\
\text { (2016a) }\end{array}$ \\
\hline & Aspergillus flavipes & $\mathrm{IC}_{50}: 55 \mu \mathrm{M}$ & $\begin{array}{l}\text { Wang et al. } \\
(2016)\end{array}$ \\
\hline \multirow[t]{2}{*}{ Methyl dichloroasterrate (154), } & Aspergillus flavipes & $\mathrm{IC}_{50}: 1.45 \mathrm{mM}$ & $\begin{array}{l}\text { Zhang et al. } \\
\text { (2016a) }\end{array}$ \\
\hline & Aspergillus flavipes & $\begin{array}{l}\mathrm{IC}_{50}: 90 \mu \mathrm{M} ; K_{\mathrm{i}}: \\
\quad 2.8 \mu \mathrm{M}\end{array}$ & $\begin{array}{l}\text { Wang et al. } \\
\text { (2016) }\end{array}$ \\
\hline 2,4-Dichloroasterric acid (155) & Aspergillus flavipes & $\mathrm{IC}_{50}: 0.091 \mathrm{mM}$ & $\begin{array}{l}\text { Zhang et al. } \\
\text { (2016a) }\end{array}$ \\
\hline 3-de-O-methylsulochrin (156) & Aspergillus flavipes & $\mathrm{IC}_{50}: 0.19 \mathrm{mM}$ & $\begin{array}{l}\text { Zhang et al. } \\
\text { (2016a) }\end{array}$ \\
\hline $\begin{array}{l}\text { 2-(3,5-Dichloro-2,6-dihydroxy-4-methylbenzoyl)-5-hydroxy-3- } \\
\text { methoxybenzoic acid (157) }\end{array}$ & Aspergillus flavipes & $\mathrm{IC}_{50}: 0.042 \mathrm{mM}$ & $\begin{array}{l}\text { Zhang et al. } \\
\text { (2016a) }\end{array}$ \\
\hline Monomethylosoic acid (158) & Aspergillus flavipes & $\mathrm{IC}_{50}: 9.9 \mu \mathrm{M}$ & $\begin{array}{l}\text { Wang et al. } \\
\text { (2016) }\end{array}$ \\
\hline Chrysines B (159) & $\begin{array}{l}\text { Penicillium } \\
\text { chrysogenum }\end{array}$ & $\mathrm{IC}_{50}: 0.35 \mathrm{mM}$ & $\begin{array}{l}\text { Wang et al. } \\
\text { (2018) }\end{array}$ \\
\hline Chrysines C (160) & $\begin{array}{l}\text { Penicillium } \\
\text { chrysogenum }\end{array}$ & $\mathrm{IC}_{50}: 0.20 \mathrm{mM}$ & $\begin{array}{l}\text { Wang et al. } \\
\text { (2018) }\end{array}$ \\
\hline Methyl-3'-methoxy-3,5-dichloroasterric acid (161) & $\begin{array}{l}\text { Penicillium } \\
\text { chrysogenum }\end{array}$ & $\mathrm{IC}_{50}: 0.15 \mathrm{mM}$ & $\begin{array}{l}\text { Wang et al. } \\
\text { (2018) }\end{array}$ \\
\hline Methyl chloroasterrate (162) & $\begin{array}{l}\text { Penicillium } \\
\text { chrysogenum }\end{array}$ & $\mathrm{IC}_{50}: 0.14 \mathrm{mM}$ & $\begin{array}{l}\text { Wang et al. } \\
\quad(2018)\end{array}$ \\
\hline Mono-chlorosulochrin (163) & $\begin{array}{l}\text { Penicillium } \\
\text { chrysogenum }\end{array}$ & $\mathrm{IC}_{50}: 0.14 \mathrm{mM}$ & $\begin{array}{l}\text { Wang et al. } \\
\text { (2018) }\end{array}$ \\
\hline Compound 164 & Aspergillus flavus & $\mathrm{IC}_{50}: 165.2 \mu \mathrm{M}$ & $\begin{array}{l}\text { Wu et al. } \\
\text { (2018) }\end{array}$ \\
\hline Compound 165 & Aspergillus flavus & $\mathrm{IC}_{50}: 129.9 \mu \mathrm{M}$ & $\begin{array}{l}\text { Wu et al. } \\
\text { (2018) }\end{array}$ \\
\hline Peniciaculin A (166) & Aspergillus flavus & $\mathrm{IC}_{50}: 1.5 \mu \mathrm{M}$ & $\begin{array}{l}\text { Wu et al. } \\
\text { (2018) }\end{array}$ \\
\hline Expansol D (167) & Aspergillus flavus & $\mathrm{IC}_{50}: 2.3 \mu \mathrm{M}$ & $\begin{array}{l}\text { Wu et al. } \\
\text { (2018) }\end{array}$ \\
\hline Dichloroorcinol (168) & $\begin{array}{l}\text { Penicillium } \\
\text { chrysogenum }\end{array}$ & $\mathrm{IC}_{50}: 0.16 \mathrm{mM}$ & $\begin{array}{l}\text { Wang et al. } \\
\text { (2018) }\end{array}$ \\
\hline Daldinione E (169) & $\begin{array}{l}\text { Daldinia } \\
\text { eschscholtzii }\end{array}$ & $\mathrm{IC}_{50}: 54 \mu \mathrm{M}$ & $\begin{array}{l}\text { Liao et al. } \\
\text { (2019) }\end{array}$ \\
\hline Bacillisporin A (170) & $\begin{array}{r}\text { Penicillium } \\
\text { aculeatum }\end{array}$ & $\mathrm{IC}_{50}: 95.8 \mu \mathrm{M}$ & $\begin{array}{l}\text { Huang et al. } \\
\text { (2017) }\end{array}$ \\
\hline Bacillisporin B (171) & $\begin{array}{r}\text { Penicillium } \\
\text { aculeatum }\end{array}$ & $\mathrm{IC}_{50}: 33.5 \mu \mathrm{M}$ & $\begin{array}{l}\text { Huang et al. } \\
\text { (2017) }\end{array}$ \\
\hline $6^{\prime}$-Methyl-[1,1'-biphenyl]-3,3', $4^{\prime}, 5$-tetraol (172) & Penicillium sp. & $\mathrm{IC}_{50}: 2.2 \mu \mathrm{M}$ & $\begin{array}{l}\text { Liu et al. } \\
\text { (2015) }\end{array}$ \\
\hline$( \pm)$-Penifupyrone (173) & Penicillium sp. & $\mathrm{IC}_{50}=14.4 \mu \mathrm{M}$ & $\begin{array}{l}\text { Liu et al. } \\
\text { (2015) }\end{array}$ \\
\hline Flaviphenalenone B (174) & Aspergillus flavipes & $\left.\mathrm{IC}_{50}: 94.9 \mu \mathrm{M}\right)$ & $\begin{array}{l}\text { Zhang et al. } \\
\text { (2016b) }\end{array}$ \\
\hline
\end{tabular}


Table 6 continued

\begin{tabular}{|c|c|c|c|}
\hline Compd. & Source & $\begin{array}{l}\alpha \text {-Glucosidase } \\
\text { activity }\end{array}$ & References \\
\hline Flaviphenalenone C (175) & Aspergillus flavipes & $\mathrm{IC}_{50}: 78.9 \mu \mathrm{M}$ & $\begin{array}{l}\text { Zhang et al. } \\
\text { (2016b) }\end{array}$ \\
\hline Cryptosporioptide (176) & $\begin{array}{l}\text { Cryptosporiopsis } \\
\text { sp. }\end{array}$ & $\mathrm{IC}_{50}: 50.5 \mu \mathrm{M}$ & $\begin{array}{l}\text { Tousif et al. } \\
\text { (2014) }\end{array}$ \\
\hline Cryptosporioptide A (177) & $\begin{array}{l}\text { Cryptosporiopsis } \\
\text { sp. }\end{array}$ & $\mathrm{IC}_{50}: 44.9 \mu \mathrm{M}$ & $\begin{array}{l}\text { Tousif et al. } \\
\text { (2014) }\end{array}$ \\
\hline Cryptosporioptide B (178) & $\begin{array}{l}\text { Cryptosporiopsis } \\
\text { sp. }\end{array}$ & $\mathrm{IC}_{50}: 41.2 \mu \mathrm{M}$ & $\begin{array}{l}\text { Tousif et al. } \\
\text { (2014) }\end{array}$ \\
\hline Compound 179 & Aspergillus flavus & $\mathrm{IC}_{50}: 4.5 \mu \mathrm{M}$ & $\begin{array}{l}\text { Wu et al. } \\
\text { (2018) }\end{array}$ \\
\hline Compound 180 & Aspergillus flavus & $\mathrm{IC}_{50}: 3.1 \mu \mathrm{M}$ & $\begin{array}{l}\text { Wu et al. } \\
\text { (2018) }\end{array}$ \\
\hline Aecilodepsipeptide A (181) & $\begin{array}{l}\text { Paecilomyces } \\
\text { formosus }\end{array}$ & $\mathrm{IC}_{50}: 74.2 \mu \mathrm{g} / \mathrm{mL}$ & $\begin{array}{l}\text { Bilal et al. } \\
\text { (2018) }\end{array}$ \\
\hline YW3548 (182) & $\begin{array}{l}\text { Paecilomyces } \\
\text { formosus }\end{array}$ & $\mathrm{IC}_{50}: 61.8 \mu \mathrm{g} / \mathrm{mL}$ & $\begin{array}{l}\text { Bilal et al. } \\
\text { (2018) }\end{array}$ \\
\hline Nectriacid A (183) & Nectria sp. & $\mathrm{IC}_{50}=121.8 \mu \mathrm{M}$ & $\begin{array}{l}\text { Cui et al. } \\
\text { (2016) }\end{array}$ \\
\hline Nectriacid B (184) & Nectria sp. & $\mathrm{IC}_{50}=23.5 \mu \mathrm{M}$ & $\begin{array}{l}\text { Cui et al. } \\
\text { (2016) }\end{array}$ \\
\hline Nectriacid C (185) & Nectria sp. & $\mathrm{IC}_{50}=42.3 \mu \mathrm{M}$ & $\begin{array}{c}\text { Cui et al. } \\
\text { (2016) }\end{array}$ \\
\hline Helicascolide A (186) & $\begin{array}{l}\text { Daldinia } \\
\quad \text { eschscholtzii }\end{array}$ & $\left.\mathrm{IC}_{50}: 16 \mu \mathrm{M}\right)$ & $\begin{array}{l}\text { Liao et al. } \\
\text { (2019) }\end{array}$ \\
\hline Helicascolide B (187) & $\begin{array}{l}\text { Daldinia } \\
\text { eschscholtzii }\end{array}$ & $\mathrm{IC}_{50}: 31 \mu \mathrm{M}$ & $\begin{array}{l}\text { Liao et al. } \\
\text { (2019) }\end{array}$ \\
\hline Helicascolide D (188) & $\begin{array}{l}\text { Daldinia } \\
\quad \text { eschscholtzii }\end{array}$ & $\mathrm{IC}_{50}: 20 \mu \mathrm{M}$ & $\begin{array}{l}\text { Liao et al. } \\
\text { (2019) }\end{array}$ \\
\hline Helicascolide E (189) & $\begin{array}{l}\text { Daldinia } \\
\text { eschscholtzii }\end{array}$ & $\mathrm{IC}_{50}: 240 \mu \mathrm{M}$ & $\begin{array}{l}\text { Liao et al. } \\
\text { (2019) }\end{array}$ \\
\hline
\end{tabular}

ranging from 0.042 to $1.47 \mathrm{mM}$ (Table 6). Among these tested metabolites, compounds $157\left(\mathrm{IC}_{50}\right.$ : $0.042 \mathrm{mM}$ ) was the most active followed by $\mathbf{1 5 5}$ $\left(\mathrm{IC}_{50}: 0.091 \mathrm{mM}\right)$ and their effects were higher than acarbose ( $\mathrm{IC}_{50}: 0.685 \mathrm{mM}$ ) (Zhang et al. 2016a). In another investigation of the fungus A. flavipes, compounds 153 and 154 along with monomethylosoic acid (158) were also isolated (Wang et al. 2016). Compounds $153\left(\mathrm{IC}_{50}: 55 \mu \mathrm{M}\right)$ and $154\left(\mathrm{IC}_{50}: 90 \mu \mathrm{M}\right)$ along with monomethylosoic acid $\left(\mathbf{1 5 8} ; \mathrm{IC}_{50}: 9.9 \mu \mathrm{M}\right)$ inhibited $\alpha$-glucosidase while a corresponding kinetic study revealed that compound $\mathbf{1 5 4}$ is a noncompetitive inhibitor with $K_{\mathrm{i}}: 2.8 \mu \mathrm{M}$ (Wang et al. 2016).
The fungus Penicillium chrysogenum produced diphenl ethers, chrysines B (159), C (160), compound 161, and methyl chloroasterrate (162), together with benzophenone, mono-chlorosulochrin (163) (Fig. 23). The group demonstrated that all these compounds illustrated $\alpha$-glucosidase effects with $\mathrm{IC}_{50}$ : ranging from $0.15 \mathrm{mM}$ to $0.35 \mathrm{mM}$ (Table 6). Most of the compounds were more potent than the standard acarbose with an $\mathrm{IC}_{50}$ of $0.28 \mathrm{mM}$ (Wang et al. 2018). The fungus Aspergillus flavus produced compounds 164-167 whose structures were established via spectroscopic techniques. Compounds $\mathbf{1 6 6}$ and 167 exhibited significant inhibition potential with $\mathrm{IC}_{50}$ values of 1.5 , and $2.3 \mu \mathrm{M}$, respectively. Furthermore, 


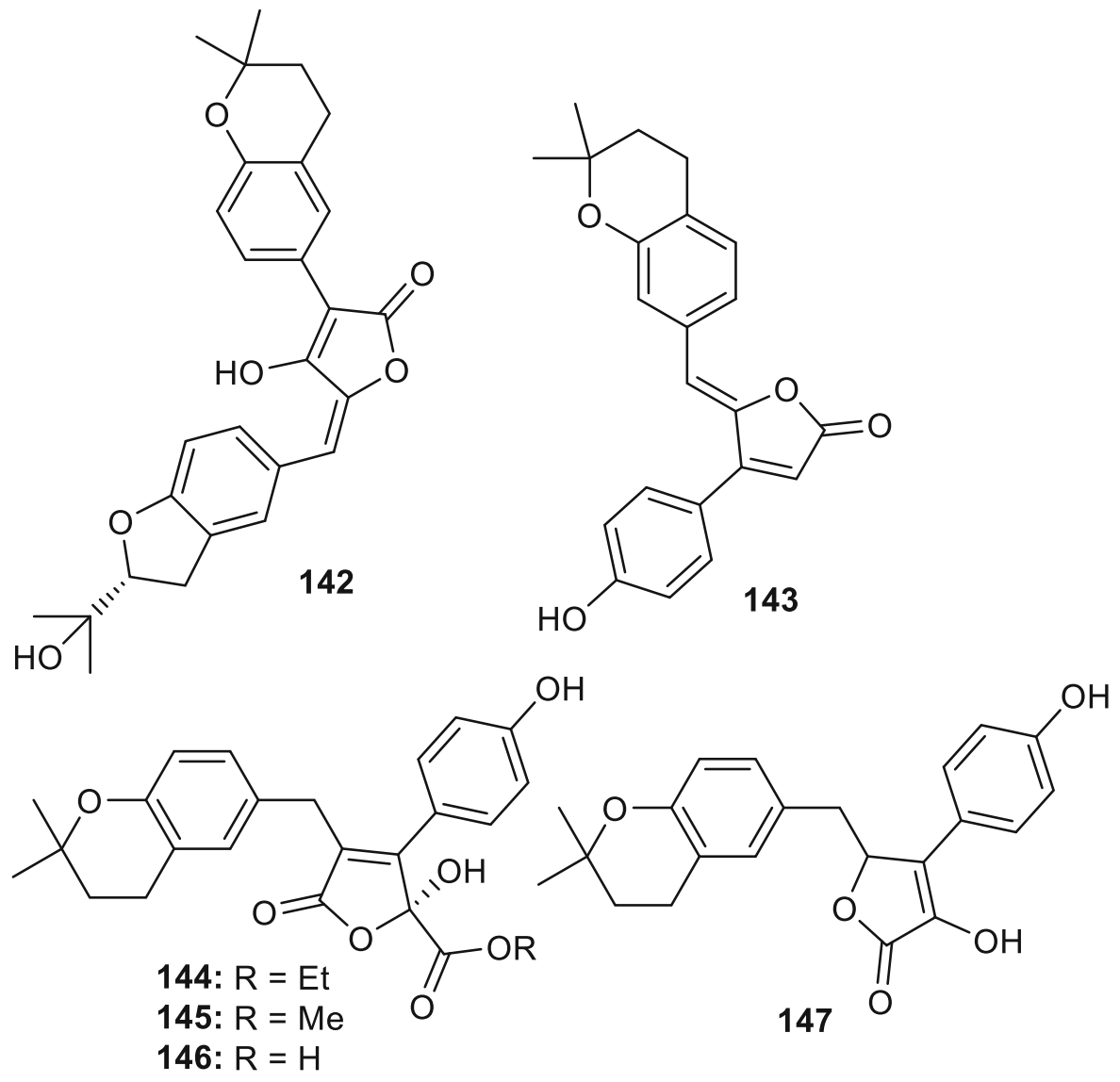

Fig. 20 Structures of butanolides 142-147

metabolites 164 and 165 were only moderately active with $\mathrm{IC}_{50}$ values of 165 and $129 \mu \mathrm{M}$, respectively. However, their effects were higher than acarbose which had an $\left.\mathrm{IC}_{50}: 840.2 \mu \mathrm{M}\right)$ (Wu et al. 2018).

\section{Miscellaneous}

The fungus Penicillium chrysogenum produces dichloroorcinol (168) (Fig. 24) which demonstrated good $\alpha$-glucosidase effects with an $\mathrm{IC}_{50}: 0.16 \mathrm{mM}$ (Wang et al. 2018). Daldinione E (169: $\left.\mathrm{IC}_{50}: 54 \mu \mathrm{M}\right)$ was obtained from the fungus Daldinia eschscholtzii and illustrated $\alpha$-glucosidase effects (Liao et al. 2019). In another investigation, bacillisporin A (170: $\mathrm{IC}_{50}$ : $95.8 \mu \mathrm{M})$ and bacillisporin $\mathrm{B}\left(\mathbf{1 7 1}: \mathrm{IC}_{50}: 33.5 \mu \mathrm{M}\right)$ were isolated from the fungus Penicillium aculeatum and both substances inhibited $\alpha$-glucosidase activity (Huang et al. 2017; Lin et al. 2008). The fungus Penicillium sp. produced compound $172 \quad\left(\mathrm{IC}_{50}\right.$ :
$2.2 \mu \mathrm{M})$ and $( \pm)$-penifupyrone $\quad\left(\mathbf{1 7 3}: \mathrm{IC}_{50-}\right.$ $=14.4 \mu \mathrm{M})$ and both compounds inhibited $\alpha$-glucosidase (Liu et al. 2015a ). Flaviphenalenones B (174: $\mathrm{IC}_{50}$ : $\left.94.9 \mu \mathrm{M}\right)$ and $\mathrm{C}\left(\mathbf{1 7 5}: \mathrm{IC}_{50}: 78.9 \mu \mathrm{M}\right)$ were isolated from the fungus Aspergillus flavipes and illustrated anti- $\alpha$-glucosidase effects (Table 6). Moreover, these compounds demonstrated a greater potency than that of the acarbose $\left(\mathrm{IC}_{50}: 685 \mu \mathrm{M}\right)$ but were less effective than quercetin $\left(\mathrm{IC}_{50}: 14.5 \mu \mathrm{M}\right)$ (Zhang et al. 2016b).

Cryptosporioptide (176: $\left.\mathrm{IC}_{50}: 50.5 \mu \mathrm{M}\right)$ along with cryptosporioptide A (177: $\left.\mathrm{IC}_{50}: 44.9 \mu \mathrm{M}\right)$ and $\mathrm{B}(\mathbf{1 7 8}$ : $\mathrm{IC}_{50}: 41.2 .5 \mu \mathrm{M}$ ) (Fig. 25) were obtained from the fungus Cryptosporiopsis sp. and all three compounds inhibited $\alpha$-glucosidase with comparable activity to the standard acarbose with an $\mathrm{IC}_{50}$ value of $38.2 \mu \mathrm{M}$ (Tousif et al. 2014). The fungus Aspergillus flavus furthermore produced compounds 179 and 180 which exhibited significant potential with $\mathrm{IC}_{50}$ values of 4.5 and $3.1 \mu \mathrm{M}$, respectively. Interestingly the effects of 


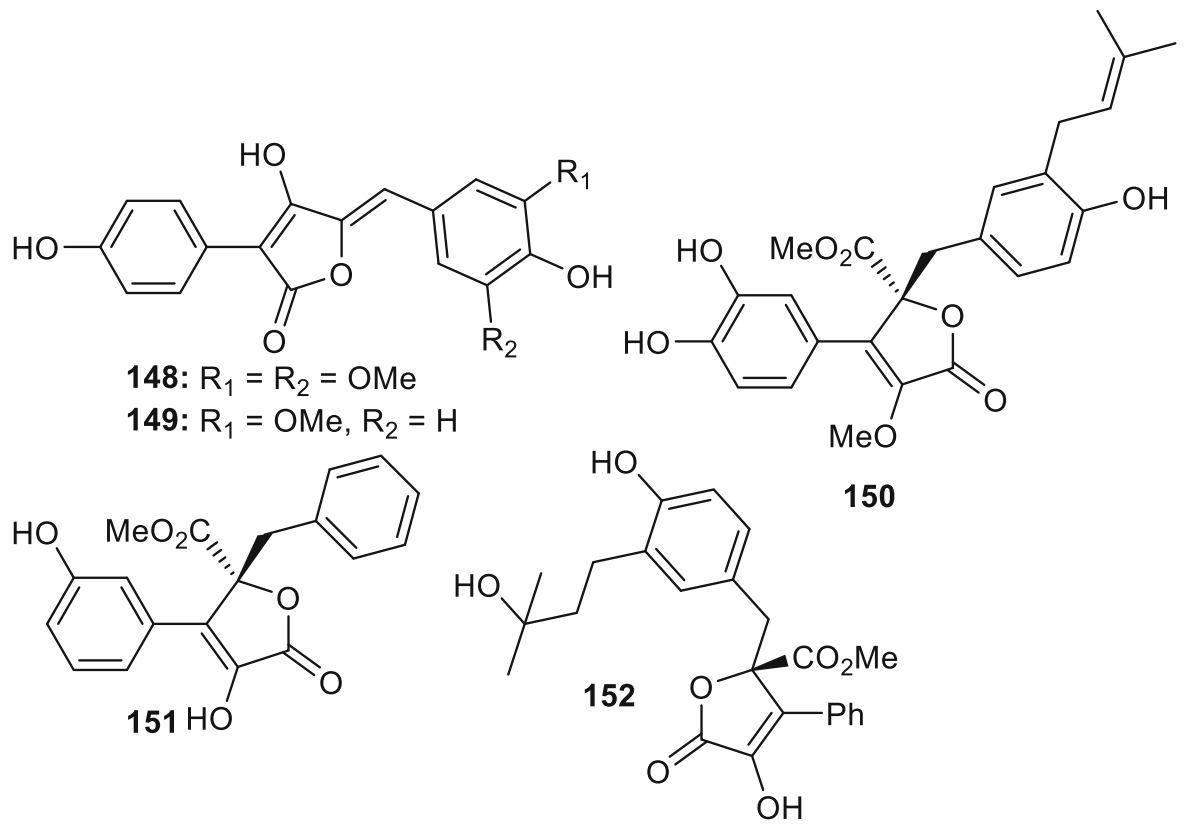

Fig. 21 Structures of butanolides 148-152

Fig. 22 Structures of butanolides 153-158<smiles>[R20]OC(=O)c1cc(O)cc(OC)c1Oc1c(Cl)c(C)c(Cl)c(O)c1C(=O)O[R2]</smiles>

153: $R_{1}=R_{2}=M e$, 154: $R_{1}=R_{2}=M e$, 155: $R_{1}=M e, R_{2}=H$<smiles>[R20]OC(=O)c1cc(O)cc([R20])c1C(=O)c1c(O)c([R3])c([R4])c([R4])c1O</smiles>

156: $R_{1}=M e, R_{2}=R_{3}=R_{4}=H$ 157: $R_{1}=H, R_{2}=M e, R_{3}=R_{4}=C l$<smiles>COc1cc(O)cc(C(=O)O)c1Oc1cc(C)cc(O)c1C(=O)O</smiles>

these compounds were higher than the reference compound acarbose $\left.\mathrm{IC}_{50}: 840.2 \mu \mathrm{M}\right)$ (Wu et al. 2018). Aecilodepsipeptide A (181: $\mathrm{IC}_{50}: 74.2 \mu \mathrm{g} /$ $\mathrm{mL})$ and $\mathrm{YW} 3548\left(\right.$ 182: $\left.\mathrm{IC}_{50}: 61.8 \mu \mathrm{g} / \mathrm{mL}\right)$ were obtained from the fungus Paecilomyces formosus and illustrated good anti- $\alpha$-glucosidase effects (Bilal et al. 2018). The polyketides, nectriacids A-C (183-185) were isolated from the fungus Nectria $\mathrm{sp}$. In this regard polyketides $184\left(\mathrm{IC}_{50}=23.5 \mu \mathrm{M}\right)$ and $185\left(\mathrm{IC}_{50}\right.$ $=42.3 \mu \mathrm{M})$ illustrated rather good $\alpha$-glucosidase 
<smiles>[R2]OC(=O)c1cc(O[R6])cc(OC)c1Oc1c([R])c(C)c([R])c(O)c1C(=O)O[R20]</smiles><smiles>COC(=O)c1cc(O)cc(OC)c1C(=O)c1c(O)cc(C)c(Cl)c1O</smiles><smiles>Cc1cc(O)cc(Oc2ccc(O)c(O)c2)c1</smiles>

159: $R_{1}=E t, R_{2}=M e, R_{3}=H, R_{4}=R_{5}=C l$

160: $R_{1}=R_{2}=R_{3}=M e, R_{4}=C l, R_{5}=H$

161: $R_{1}=R_{2}=R_{3}=M e, R_{4}=R_{5}=C l$

162: $R_{1}=R_{3}=R_{4}=H, R_{2}=M e, R_{5}=C l$<smiles>Cc1cc(O)cc(Oc2cc(C)c(C(=O)OCC(O)C(O)CO)c(O)c2)c1</smiles><smiles>COc1cc(O)cc(Oc2cc(C)cc(OCc3ccc(C(C)(O)CCCC(C)C)c(O)c3)c2)c1</smiles>

Fig. 23 Structures of butanolides 159-167<smiles>CCc1c(O)cc2c(=O)oc(C)c(C)c(=O)c2c1OC</smiles><smiles>COc1c(O)c2c3c(c(O)c(CC=C(C)C)c(C)c3c1OC)C(=O)OC2=O</smiles>

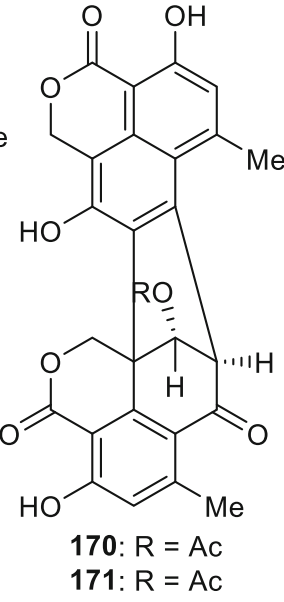

171: $R=A c$<smiles>COC(=O)c1cc(OC)cc(OC)c1C(=O)c1coc2c1OC(C)CC2=O</smiles><smiles>COc1c2c(O)c(OC)c3c(c(O)c(CC=C(C)C)c(C)c13)C(=O)C(=O)O2</smiles>

Fig. 24 Structures of butanolides 168-175

effects which were higher than acarbose $\left(\mathrm{IC}_{50-}\right.$ $=815.3 \mu \mathrm{M})$. On the other hand nectriacid $\mathrm{A}(\mathbf{1 8 3})$ also possessed good $\alpha$-glucosidase effects with $\mathrm{IC}_{50-}$ $=121.8 \mu \mathrm{M}$ (Cui et al. 2016).

Helicascolides A (186: $\mathrm{IC}_{50}$ : $\left.16 \mu \mathrm{M}\right)$, B (187: $\mathrm{IC}_{50}$ : $31 \mu \mathrm{M}), \mathrm{D}\left(\mathbf{1 8 8}: \mathrm{IC}_{50}: 20 \mu \mathrm{M}\right)$, and $\mathrm{E}\left(189: \mathrm{IC}_{50}\right.$ : $240 \mu \mathrm{M}$ ) (Fig. 26) are all produced by the fungus Daldinia eschscholtzii and these isolated compounds illustrated excellent $\alpha$-glucosidase effects (Table 7). Compound 186 was the most potent followed by compound 188 while metabolite $\mathbf{1 8 9}$ was the least active. The activity difference between compounds 188 and 189 indicated that it was indeed the acetyl group at C-8 that was responsible for the reduced activity (Liao et al. 2019). Polyketides viz., aspergones A (190), B (191), E (192), J (193), K (194), N-Q 


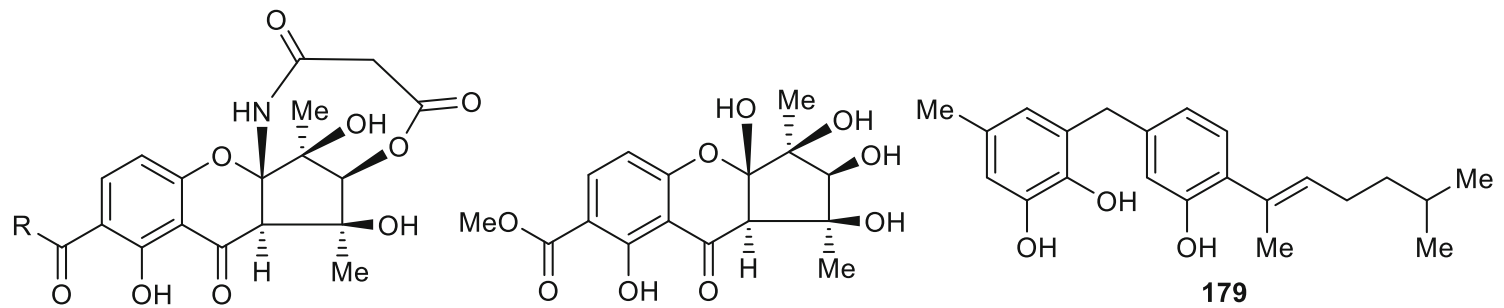

176: $\mathrm{R}=\mathrm{OMe}$

178

177: $\mathrm{R}=\mathrm{OH}$<smiles>CC(C)CCC[C@](C)(O)c1ccc(COCc2ccc([C@](C)(O)CCCC(C)C)c(O)c2)cc1O</smiles>

180

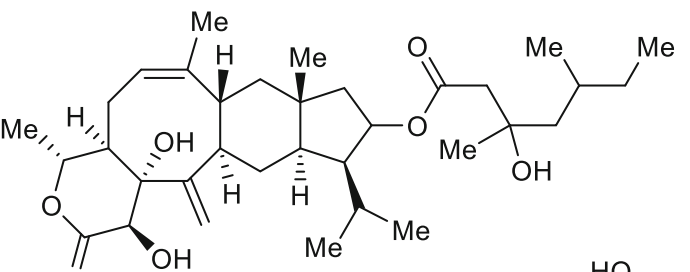

182<smiles>CC(/C=C/C(C)=C/C(C)=C/C(C)=C/C(=O)O)=C\C(=O)O</smiles>

181<smiles>[M]CC=CC=CC=CC=CC(=O)O</smiles>

Fig. 25 Structures of butanolides 176-185

(195-198) were isolated from the fungus Aspergillus sp. and all these compounds inhibited $\alpha$-glucosidase with $\mathrm{IC}_{50}$ values ranging from 1.30 to $2.37 \mathrm{mM}$ (Table 7). Moreover all the compounds were less effective than acarbose $\left(\mathrm{IC}_{50}: 0.95 \mathrm{mM}\right.$ ) (Kong et al. 2015). The fungus Penicillium expansum produced expansolides C (199) and D (200). An epimeric mixture of compounds 199 and 200 (2:1) potentially inhibited $\alpha$-glucosidase $\left(\mathrm{IC}_{50}=0.50 \mathrm{mM}\right)$ and thus was more potent than acarbose $\left(\mathrm{IC}_{50}=1.90 \mathrm{mM}\right.$ ) (Ying et al. 2017).

The fungus Ganoderma lucidum produces nortriterpenoids, (201), D (202), E (203) (Fig. 27). Metabolite 202 exerted potent $\alpha$-glucosidase effects among these compounds, with an $\mathrm{IC}_{50}$ value of $41.7 \mu \mathrm{M}$, followed by metabolites $201 \quad\left(\mathrm{IC}_{50-}\right.$ $=81.8 \mu \mathrm{M})$ and $203\left(\mathrm{IC}_{50}=91.3 \mu \mathrm{M}\right)$. All these compounds were more potent than acarbose $\left(\mathrm{IC}_{50}\right.$ : $669.7 \mu \mathrm{M})$ (Zhao et al. 2015). A pair of enantiomers: (+)-1-hydroxyboivinianic acid (204a), (-)-1-hydroxyboivinianic acid (204b) along with 7-deoxy-7,14didehydrosydonol (205) were separated from the fungus Aspergillus versicolor and evaluated for their $\alpha$-glucosidase inhibition. Metabolite $205 \quad\left(\mathrm{IC}_{50}\right.$ : $7.5 \mu \mathrm{M}$ ) was the most potent inhibitor when compared with the activity of acarbose $\left(\mathrm{IC}_{50}=350 \mu \mathrm{M}\right)$. On the other hand compounds (+)-204 $\left(\mathrm{IC}_{50}: 120.3 \mu \mathrm{M}\right)$ and $(-)-204\left(\mathrm{IC}_{50}: 113.3 \mu \mathrm{M}\right)$ are reported as moderate inhibitors (Cui et al. 2018).

The fungus Setosphaeria rostrata produced thiodiketopiperazine, exserohilone (206) (Fig. 28) and possess $\alpha$-glucosidase activity with an $\mathrm{IC}_{50}$ value of $82 \mu \mathrm{g} / \mathrm{mL}$ (Centko et al. 2017). Moreover, asperpanoid A (207) was obtained from Aspergillus sp. and 


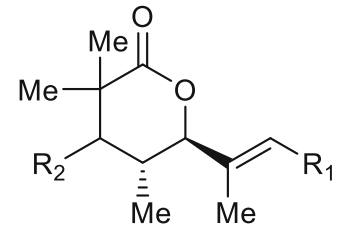

186: $\mathrm{R}_{1}=\mathrm{Me}, \mathrm{R}_{2}=\alpha-\mathrm{OH}$

187: $R_{1}=M e, R_{2}=\beta-O H$

188: $\mathrm{R}_{1}=\mathrm{CH}_{2} \mathrm{OH}, \mathrm{R}_{2}=\alpha-\mathrm{OH}$

189: $\mathrm{R}_{1}=\mathrm{CH}_{2} \mathrm{OAc}, \mathrm{R}_{2}=\alpha-\mathrm{OH}$<smiles>[R1]CC=CC=C1C=C(CC([R2])C)C(=O)O1</smiles>

190: $\mathrm{R}_{1}=\mathrm{OH}, \mathrm{R}_{2}=\mathrm{H}, \Delta^{4}-\mathrm{Z}$

191: $R_{1}=H, R_{2}=O H, \Delta^{4}-Z$<smiles>C=C/C=C\[C@H]1OC(=O)[C@@H](CCC)[C@@H]1O</smiles>

192<smiles>C=CC=CC=C[C@](O)(C=CC)CO</smiles>

193: $\Delta^{5}-E$

194: $\Delta^{5}-Z$<smiles>[R]C1C(CO)=C(CO)[C@H](/C=C/C)[C@H](O)[C@H]1O</smiles>

195: $\mathrm{R}=(R)-\mathrm{OH}, 5 \mathrm{R}$ 196: $\mathrm{R}=(S)-\mathrm{Cl}, 5 S$<smiles>C/C=C/C1=C(CO)[C@@H](O)[C@H]2O[C@H]2[C@H]1O</smiles>

197<smiles>C/C=C/C1=C(CO)[C@H](O)[C@@H](O)[C@H]2OC(=O)O[C@H]12</smiles><smiles>C=C1C(O)CC2CC1C1(CO[C@]3(C[C@H](C)C(=O)O3)C1)C2</smiles><smiles>C=C1C(O)CC2CC1C1(CO[C@]3(C[C@@H](C)C(=O)O3)O1)C2</smiles>

Fig. 26 Structures of butanolides 186-200

possesses $\alpha$-glucosidase activity with $\mathrm{IC}_{50}: 12.4 \mu \mathrm{M}$ (Cai et al. 2019). The fungus Zasmidium sp. produced tripalmitin (208: $\mathrm{IC}_{50}$ : $\left.3.75 \mu \mathrm{M}\right)$ which illustrated potent $\alpha$-glucosidase activity (Lopéz et al. 2019). Metabolites dothiorelone K (209: $\left.\mathrm{IC}_{50}: 22 \mu \mathrm{g} / \mathrm{mL}\right)$, L (210: $\left.\mathrm{IC}_{50}: 77.9 \mu \mathrm{g} / \mathrm{mL}\right)$, and I (211: $\left.\mathrm{IC}_{50}: 5.4 \mu \mathrm{g} / \mathrm{mL}\right)$ were reported from the fungus Dothiorella sp. and illustrated $\alpha$-glucosidase effects (Zheng et al. 2019).

\section{Conclusion}

Fungi are known as prolific producers of diverse secondary metabolites. Fungal metabolites range from simple small molecules to more complex systems viz., proteins and polypeptides with a wide range of biological activities. Since the scurge of diabetes has increased worldwide, it is estimated that by 2040 , the number of diabetic people will increase to 642 million around the globe. There is thus a critical need to find new antidiabetic drugs with less side effects. In spite of the fact that drug treatment for diabetes has improved over the last decade, drug resistance too has become an important issue in diabetic drug discovery and consequently new and improved strategies will have to be sought. One such strategy would be to either inhibit or decrease the manufacture of glucose in the small intestine. $\alpha$-Glucosidase inhibitors can reduce the digestion of carbohydrates and thus could be considered as one of the most effective strategies to reduce post-prandial hyperglycemia. $\alpha$ Glucosidase inhibitors are thus an important group of therapeutic agents to treat diabetes which currently comprises of three drugs viz., acarbose, miglitol, and voglibose (Derosa and Maffioli 2012). Numberous studies have been conducted to analyze the clinical efficacy and safety of acarbose, miglitol, and voglibose $\alpha$-glucosidase with respect to glycemic control, atherosclerosis, and inflammation. It has been reported that these three $\alpha$-glucosidase inhibitors are considered to be safe and effective both in monotherapy as well as in combination with additional anti-diabetic drugs (Dash et al. 2018; Derosa and Maffioli 2012).

In this context, the last decade has been noted to be a most fruitful period in isolating low molecular weight antidiabetic compounds from fungi. During this decade (2010 to 2019), more than 200 natural products have been isolated from various fungal sources and screened for their $\alpha$-glucosidase inhibitory activity. Among the alkaloids, compound $\mathbf{8}$ exhibited an $\mathrm{IC}_{50}$ value of $3.3 \mu \mathrm{M}$, which showed that the pyrolidine-2-one system coupled with a phenolic function are important functional groups for the development of antidiabetic drugs. Moreover, among 
Table 7 Fungal metabolites 190-211 as $\alpha$-glucosidase inhibitors

\begin{tabular}{|c|c|c|c|}
\hline Compd. & Source & A-Glucosidase activity & References \\
\hline Aspergone A (190) & Aspergillus sp. & $\mathrm{IC}_{50}=2.36 \mathrm{mM}$ & Kong et al. (2015) \\
\hline Aspergone B (191) & Aspergillus sp. & $\mathrm{IC}_{50}=1.65 \mathrm{mM}$ & Kong et al. (2015) \\
\hline Aspergone E (192) & Aspergillus sp. & $\mathrm{IC}_{50}=1.30 \mathrm{mM}$ & Kong et al. (2015) \\
\hline Aspergone J (193) & Aspergillus sp. & $\mathrm{IC}_{50}=2.37 \mathrm{mM}$ & Kong et al. (2015) \\
\hline Aspergone K (194) & Aspergillus sp. & $\mathrm{IC}_{50}=2.70 \mathrm{mM}$ & Kong et al. (2015) \\
\hline Aspergone N (195) & Aspergillus sp. & $\mathrm{IC}_{50}=1.36 \mathrm{mM}$ & Kong et al. (2015) \\
\hline Aspergone O (196) & Aspergillus sp. & $\mathrm{IC}_{50}=1.54 \mathrm{mM}$ & Kong et al. (2015) \\
\hline Aspergone P (197) & Aspergillus sp. & $\mathrm{IC}_{50}=2.21 \mathrm{mM}$ & Kong et al. (2015) \\
\hline Aspergone Q (198) & Aspergillus sp. & $\mathrm{IC}_{50}=2.26 \mathrm{mM}$ & Kong et al. (2015) \\
\hline Expansolide C (199) & Penicillium expansum & $\mathrm{IC}_{50}=0.50 \mathrm{mM}$ (mixture of 199 and 200) & Ying et al. (2017) \\
\hline Expansolide D (200) & Penicillium expansum & & Ying et al. (2017) \\
\hline Ganoderlactone B (201) & Ganoderma lucidum & $\mathrm{IC}_{50}: 81.8 \mu \mathrm{M}$ & Zhao et al. (2015) \\
\hline Ganoderlactone D (202) & Ganoderma lucidum & $\mathrm{IC}_{50}: 41.7 \mu \mathrm{M}$ & Zhao et al. (2015) \\
\hline Ganoderlactone E (203) & Ganoderma lucidum & $\mathrm{IC}_{50}: 91.3 \mu \mathrm{M}$ & Zhao et al. (2015) \\
\hline (+)-1-Hydroxyboivinianic acid (204a) & Aspergillus versicolor & $\mathrm{IC}_{50}: 120.3 \mu \mathrm{M}$ & Cui et al. (2018) \\
\hline$(-)-1-H y d r o x y b o i v i n i a n i c ~ a c i d ~(204 b)$ & Aspergillus versicolor & $\mathrm{IC}_{50}: 113.3 \mu \mathrm{M}$ & Cui et al. (2018) \\
\hline 7-Deoxy-7,14-didehydrosydonol (205) & Aspergillus versicolor & $\mathrm{IC}_{50}: 7.5 \mu \mathrm{M}$ & Cui et al. (2018) \\
\hline exserohilone (206) & Setosphaeria rostrata & $\mathrm{IC}_{50}: 82 \mu \mathrm{g} / \mathrm{mL}$ & Centko et al. (2017) \\
\hline Asperpanoid A (207) & Aspergillus sp. & $\mathrm{IC}_{50}: 12.4 \mu \mathrm{M}$ & Cai et al. (2019) \\
\hline Tripalmitin (208) & Zasmidium sp. & $\mathrm{IC}_{50}: 3.75 \mu \mathrm{M}$ & Lopéz et al. (2019) \\
\hline Dothiorelone K (209) & Dothiorella sp. & $\mathrm{IC}_{50}: 22 \mu \mathrm{g} / \mathrm{mL}$ & Zheng et al. (2019) \\
\hline Dothiorelone L (210) & Dothiorella sp. & $\mathrm{IC}_{50}: 77.9 \mu \mathrm{g} / \mathrm{mL}$ & Zheng et al. (2019) \\
\hline Dothiorelone I (211) & Dothiorella sp. & $\mathrm{IC}_{50}: 5.4 \mu \mathrm{g} / \mathrm{mL}$ & Zheng et al. (2019) \\
\hline
\end{tabular}

Fig. 27 Structures of butanolides 201-205

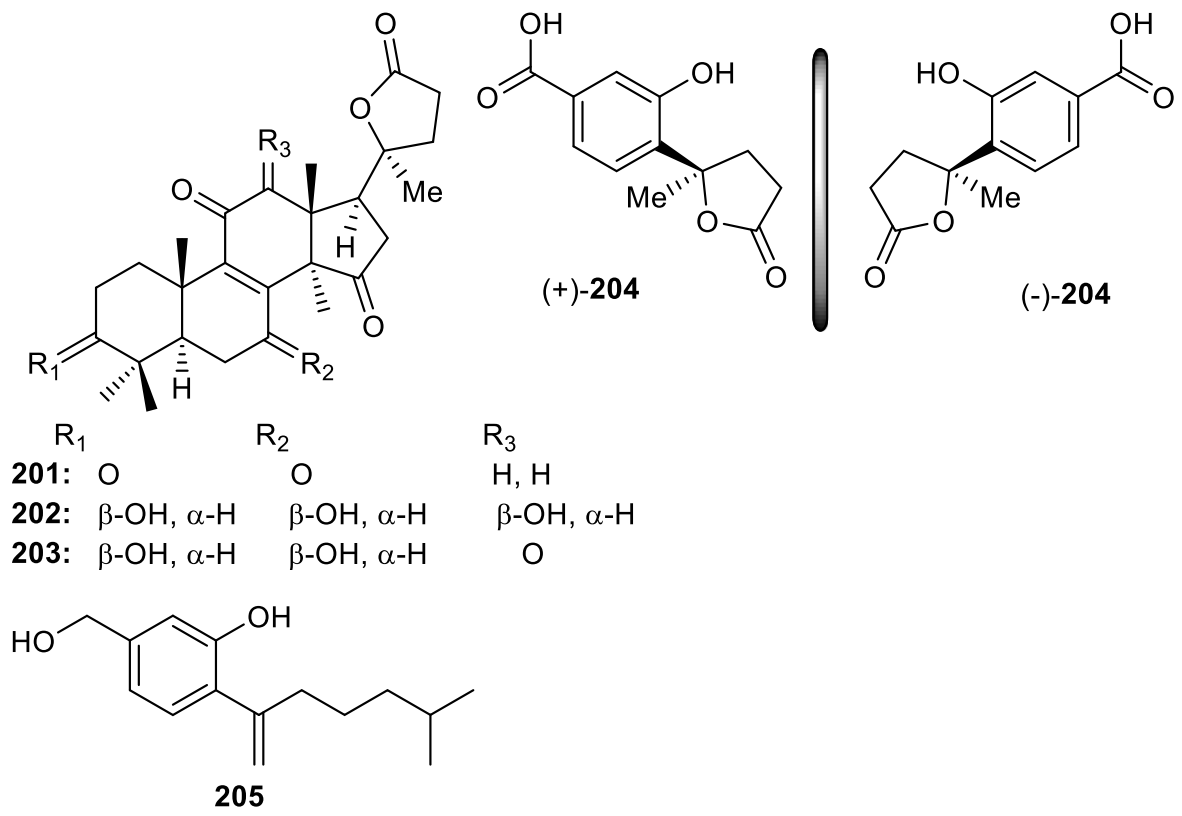


<smiles>CS[C@]12C[C@@H]3C(=O)C=C[C@H](O)[C@H]3N1C(=O)[C@@]1(SC)C[C@@]3(C)C(=O)C=C[C@H](O)[C@H]3N1C2=O</smiles>

206<smiles>CCCC(=O)OCC(COC(=O)CC)OC(=O)CCC</smiles>

Fig. 28 Structures of butanolides 206-211

p-terphenyls as $\alpha$-glucosidase inhibitors, sarcoviolin $\beta$ (23, $\left.\quad \mathrm{IC}_{50}=0.58 \mu \mathrm{M}\right), \quad 3,3^{\prime \prime}$-dihydroxy-6'-Odesmethylterphenyllin $\left(\mathbf{2 2}, \mathrm{IC}_{50}=0.9 \mu \mathrm{M}\right)$ and concrescenin $\mathrm{A}\left(33, \mathrm{IC}_{50}: 0.9 \mu \mathrm{M}\right)$ were found to be the most potent $\alpha$-glucosidase inibitors. Depsides $\mathbf{4 3 - 4 5}$ showed $\alpha$-glucosidase inibiton with $\mathrm{IC}_{50}: 3-7.6 \mu \mathrm{M}$, and thus offer their strong candidature to be studied further for development as antidiabetic drugs. Among the depsidones, botryorhodine $\mathrm{D}\left(\mathbf{6 2}, \mathrm{IC}_{50}=2.1 \mu \mathrm{M}\right)$ has been identified as a potential $\alpha$-glucosidase inhibitor. Since two more depsides (63 and 64) also showed significant inhibtion, this fact revealed that the dioxipanone system is important and is also supported by other functional groups attached at C-3 to improve the $\alpha$-glucosidase activity. A reasonable number (25) of isocoumarins have been idetified from various fungi as $\alpha$-glucosidase inhibitors. Notably compound $\mathbf{9 3}$ $\left(\mathrm{IC}_{50}: 0.027 \mathrm{mM}\right)$ was found to be 35 fold more potent than the standard drug acarbose which indicated that a substituted tetrahydrofuran system might be playing a key role in the inhibitory activity. Among the benzofurans, 6-demethylpenisimplicissin $\mathbf{( 1 0 8}, \mathrm{IC}_{50}$ : $9.5 \mu \mathrm{M})$ and $1^{\prime \prime}$-epihydroxydihydrovermistatin (109, $\left.\mathrm{IC}_{50}: 8.0 \mu \mathrm{M}\right)$ have also shown their potential as antidiabetic drug candidates. However, among the few quinone exmples, only compound $115\left(\mathrm{IC}_{50-}\right.$ $=7.2 \mu \mathrm{M})$ exerted potential inhibition of $\alpha$-glucosidase being higher than the positive control genistein $\left(\mathrm{IC}_{50}=13.6 \mu \mathrm{M}\right)$, whereas, chromone analogs 125 $\left(\mathrm{IC}_{50}: 13 \mu \mathrm{M}\right)$ and $\mathbf{1 2 8}\left(\mathrm{IC}_{50}: 15 \mu \mathrm{M}\right)$ proved to be the

most active metabolites. Butanolide polyketides are another major class identified as antidiabetic fungal metabolites. A total of 29 compounds forming this group were isolated from various fungi during the last decade and almost all showed remarkable $\alpha$-glucosidase inhibitory activity. Rubrolide $\mathrm{S}\left(\mathbf{1 4 3}, \mathrm{IC}_{50-}\right.$ $=1.2 \mu \mathrm{M}$ ) with a $K \mathrm{i}$ value of $1.42 \mu \mathrm{M}$ has been identified as the most potent inhibitor. Further studies on this compound may lead to the development of a new and novel antidiabetic agent. Among the diphenyl ether dervatives, peniciaculin $\mathrm{A}\left(\mathbf{1 6 6}, \mathrm{IC}_{50}: 1.5 \mu \mathrm{M}\right)$ and expansol $\mathrm{D}\left(\mathbf{1 6 7}, \mathrm{IC}_{50}: 2.3 \mu \mathrm{M}\right)$ showed their potential as future drug candidates to treat diabetes mellitus. Other than these metabolites, 6'-Methyl[1,1'-biphenyl]-3,3', $4^{\prime}, 5$-tetraol $\left(\mathbf{1 7 2}, \mathrm{IC}_{50}: 2.2 \mu \mathrm{M}\right)$, meroterpenoids $179\left(\mathrm{IC}_{50}: 4.5 \mu \mathrm{M}\right)$ and $180\left(\mathrm{IC}_{50}\right.$ : $3.1 \mu \mathrm{M})$, sesquiterpenoid; 7-deoxy-7,14-didehydrosydonol $\left(\mathbf{2 0 5}, \mathrm{IC}_{50}: 7.5 \mu \mathrm{M}\right)$, tripalmitin $\left(\mathbf{2 0 8}, \mathrm{IC}_{50}\right.$ : $3.75 \mu \mathrm{M})$ and dothiorelone I $\left(\mathbf{2 1 1}, \mathrm{IC}_{50}: 5.4 \mu \mathrm{g} / \mathrm{mL}\right)$ are also considered as powerful candidates as $\alpha$ glucosidase inhibitors. The above information clearly demonstrates that fungi are one of the most vital sources of novel substances with diverse structural features, which can be further explored as new and noval antidiabetic agents.

Acknowledgements The author Hidayat Hussain (HH) thanks the Alexander von Humboldt Foundation for its generous support in providing the opportunity to work in Germany which facilitated the writing of this review.

Funding Open Access funding enabled and organized by Projekt DEAL..

Open Access This article is licensed under a Creative Commons Attribution 4.0 International License, which permits use, sharing, adaptation, distribution and reproduction in any medium or format, as long as you give appropriate credit to the original author(s) and the source, provide a link to the Creative Commons licence, and indicate if changes were made. The images or other third party material in this article are included in the article's Creative Commons licence, unless indicated otherwise in a credit line to the material. If material is not included in the article's Creative Commons licence and your intended use is not permitted by statutory regulation or exceeds the permitted use, you will need to obtain permission directly from the copyright holder. To view a copy of this licence, visit http://creativecommons.org/licenses/by/4.0/. 


\section{References}

Abbas G, Ahmed Al-Harrasic A, Hussain H et al (2019) The management of diabetes mellitus-imperative role of natural products against dipeptidyl peptidase- $4, \alpha$-glucosidase and sodium-dependent glucose co-transporter 2 (SGLT2). Bioorg Chem 86:305-315

Abdou R, Scherlach K, Dahse HM et al (2010) Botryorhodines A-D, antifungal and cytotoxic depsidones from Botryosphaeria rhodina, an endophyte of the medicinal plant Bidens pilosa. Phytochemistry 71:110-116

Agosti G, Birkinshaw JH, Chaplen P (1962) Studies in the biochemistry of micro-organisms. 112. Anthraquinone pigments of strains of Cladosporium fulvum Cooke. Biochem J 85:528-530

Aldridge DC, Galt S, Turner WB (1971) Metabolites of Lasiodiplodia theobromae. J Chem Soc 113:1623-1627

Anke H, Kolthoum I, Laatsch H (1980) Metabolic products of microorganisms. 192. The anthraquinones of the Aspergillus glaucus group. II. Biological activity. Arch Microbiol 126:231-236

Arai S, Wakana D, Itabashi T et al (2017) New two pebrolide derivatives, 14-deacetoxy-1-deoxypebrolide and 7'-hydroxyasperphenamate Isolated from Penicillium sp. IFM62525. Heterocycles 94(2):326-333

Askenazi M, Driggers EM, Holtzman DA et al (2003) Integrating transcriptional and metabolite profiles to direct the engineering of lovastatin-producing fungal strains. Nat Biotechnol 21:150-156

Baba K, Xiao YQ, Taniguchi M et al (1991) Isocoumarins from Coriandrum sativum. Phytochemistry 30:4143-4146

Belofsky GN, Gloer KB, Gloer JB et al (1998) New p-terphenyl and polyketide metabolites from the sclerotia of Penicillium raistrickii. J Nat Prod 61:1115-1119

Bernier D, Moser F, Brückner R (2007) Synthesis and cyclization of 3-aryl-2-(arylacetoxy)acrylates: a three-step access to pulvinones. Synthesis 15:2240-2248

Bhatia A, Singh B, Arora R et al (2019) In vitro evaluation of the $\alpha$-glucosidase inhibitory potential of methanolic extracts of traditionally used antidiabetic plants. BMC Complement Altern Med 19:74

Bilal S, Ali L, Khan AL et al (2018) Endophytic fungus Paecilomyces formosus LHL10 produces sester-terpenoid YW3548 and cyclic peptide that inhibit urease and $\alpha$-glucosidase enzyme activities. Arch Microbiol 200:1493-1502

Buayairaksa M, Kanokmedhakul S, Kanokmedhakul K et al (2011) Cytotoxic lasiodiplodin derivatives from the fungus Syncephalastrum racemosum. Arch Pharmacal Res 34:2037-2041

Cai R, Chen S, Long Y et al (2017) Depsidones from Talaromyces stipitatus SK-4, an endophytic fungus of the mangrove plant Acanthus ilicifolius. Phytochem Lett 20:196-199

Cai R, Wu Y, Chen S et al (2018) Peniisocoumarins A-J: isocoumarins from Penicillium commune QQF-3, an Endophytic Fungus of the Mangrove Plant Kandelia candel. J Nat Prod 81:1376-1383
Cai R, Jiang H, Zang Z et al (2019) New Benzofuranoids and Phenylpropanoids from the Mangrove Endophytic Fungus, Aspergillus sp. ZJ-68. Mar Drugs 17:478

Capon RJ (2020) Extracting value: mechanistic insights into the formation of natural product artifacts-case studies in marine natural products. Nat Prod Rep 37:55-79

Centko RM, Ratnaweera PB, Tysoe C et al (2017) Alpha-glucosidase and alpha-amylase inhibiting thiodiketopiperazines from the endophytic fungus Setosphaeria rostrata isolated from the medicinal plant Costus speciosus in Sri Lanka. Phytochem Lett 22:76-80

Chan CW, Yu CL, Lin JC et al (2018) Glitazones and alphaglucosidase inhibitors as the second-line oral anti-diabetic agents added to metformin reduce cardiovascular risk in Type 2 diabetes patients: a nationwide cohort observational study. Cardiovasc Diabetol 17(1):20

Chen B, Shen Q, Zhu X et al (2014) The anthraquinone derivatives from the fungus Alternaria sp. XZSBG-1 from the Saline Lake in Bange, Tibet. China. Molecules 19:16529-16542

Chen S, Liu Z, Liu Y et al (2015a) New depsidones and isoindolinones from the mangrove endophytic fungus Meyerozyma guilliermondii (HZ-Y2) isolated from the South China Sea. Beilstein J Org Chem 11:1187-1193

Chen S, Liu Z, Li H et al (2015b) $\beta$-Resorcylic acid derivatives with $\alpha$-glucosidase inhibitory activity from Lasiodiplodia sp. ZJ-HQ 1, an endophytic fungus in the medicinal plant Acanthus Ilicifolius. Phytochem Lett 13:141-146

Chen S, Liu Y, Liu Z et al (2016) Isocoumarins and benzofurans from the mangrove endophytic fungus Talaromyces amestolkiae possess a-glucosidase inhibitory and antibacterial activities. RSC Adv 6:26412-26420

Chen S, Liu Z, Liu H et al (2017) Lasiodiplactone A, a novel lactone from the mangrove endophytic fungus $\mathrm{La}$ siodiplodia theobromae ZJ-HQ1. Org Biomol Chem 15:6338-6341

Chen Y, Yang W, Zou G et al (2019) Bioactive polyketides from the mangrove endophytic fungi Phoma sp. SYSU-SK-7. Fitoterapia 139:104369

Couch RD, Gaucher GM (2004) Rational elimination of Aspergillus terreus sulochrin production. J Biotechnol 108:171-178

Cui H, Liu Y, Nie Y et al (2016) Polyketides from the mangrovederived endophytic fungus Nectria sp. HN001 and their $\alpha$ glucosidase inhibitory activity. Mar Drugs 14:86

Cui H, Liu Y, Li T et al (2018) 3-Arylisoindolinone and sesquiterpene derivatives from the mangrove endophytic fungi Aspergillus versicolor SYSU-SKS025. Fitoterapia 124:177-181

Dash RP, Babu RJ, Srinivas NR (2018) Reappraisal and perspectives of clinical drug-drug interaction potential of $\alpha$ glucosidase inhibitors such as acarbose, voglibose and miglitol in the treatment of type 2 diabetes mellitus. Xenobiotica 48:89-108

Derosa G, Maffioli P (2012) $\alpha$-Glucosidase inhibitors and their use in clinical practice. Arch Med Sci 8:899-906

Frisvad JC, Houbraken J, Popma S et al (2013) Two new Penicillium species, Penicillium buchwaldii and Penicillium spathulatum, producing the anticancer compound asperphenamate Quick View Other Sources FEMS. Microbiol Lett 339(2):77-92 
Gao JM, Yang SX, Qin JC (2013) Azaphilonoids: chemistry and biology. Chem Rev 113:4755-4811

Ge HM, Shen Y, Zhu CH et al (2008) Penicidones A-C, three cytotoxic alkaloidal metabolites of an endophytic Penicillium sp. Phytochemistry 69(2):571-576

Ghosh AC, Manmade A, Demain AL (1977) Toxins from Penicillium islandicum Sopp. In: Rodricks JV, Hesseltine $\mathrm{CW}$, Mehlman MA (eds) Mycotoxins in human and animal health. Pathotox, Chicago, pp 625-638

Hampl V, Wetzel I, Bracher F et al (2011) New substituted isocoumarins and dihydroisocoumarins and their cytotoxic activities. Sci Pharm 79:21-30

Hargreaves J, Park J, Ghisalberti E et al (2002) New chlorinated diphenyl ethers from an Aspergillus species. J Nat Prod 65:7-10

He F, Li X, Yu JH et al (2019) Secondary metabolites from the mangrove sediment-derived fungus Penicillium pinophilum SCAU037. Fitoterapia 136:104177

Hossain MA, Pervin R (2018) Current antidiabetic drugs: review of their efficacy and safety, 2nd edn. In: Nutritional and therapeutic interventions for diabetes and metabolic syndrome. Elsevier, Academic Press, Waltham, pp 455-473

Hou XM, Zhang YH, Hai Y et al (2017) Aspersymmetide A, a new centrosymmetric cyclohexapeptide from the marinederived fungus Aspergillus versicolor. Mar Drugs 15(11):363/1-363/8

Huang H, Feng X, Xiao Z et al (2011) Azaphilones and p-terphenyls from the mangrove endophytic fungus Penicillium chermesinum (ZH4-E2) isolated from the South China Sea. J Nat Prod 74:997-1002

Huang H, Liu T, Wu X et al (2017) A new antibacterial chromone derivative from mangrove-derived fungus Penicillium aculeatum (No. 9 EB). Nat Prod Res 31(22):2593-2598

Hung HY, Qian K, Morris-Natschke SL et al (2012) Recent discovery of plant-derived anti-diabetic natural products. Nat Prod Rep 29:580-606

Izhaki I (2002) Emodin-a secondary metabolite with multiple ecological functions in higher plants. New Phytol 155:205-217

Jongrungruangchok S, Aree T, Sureram S et al (2013) Crystal structure of 2-(2-carboxy-4-hydroxy-6-methoxyphenoxy)3,5-dichloro-6-hydroxy-4-methylbenzoic Acid 1-methyl ester. Chin J Struct Chem 32:1742

Kakinuma N, Iwai H, Takahashi S et al (2000) Quinolactacins A, B and C: novel quinolone compounds from Penicillium sp. EPF-6. I. Taxonomy, production, isolation and biological properties. J Antibiot 53(11):1247-1251

Kao CC, Wu PC, Wu CH et al (2016) Risk of liver injury after $\alpha$ glucosidase inhibitor therapy in advanced chronic kidney disease patients. Sci Rep 6:18996

Kobayashi MI, Aoyagi A, Tanaka I et al (2008) Sterenin A, B, C and $\mathrm{D}$, novel $11 \mathrm{~b}$-hydroxysteroid dehydrogenase type 1 inhibitors from Stereum sp SANK 21205. J Antibiot 61(3):128-135

Kong F, Zhao C, Hao J et al (2015) New a-glucosidase inhibitors from a marine sponge-derived fungus, Aspergillus sp. OUCMDZ-1583. RSC Adv 5:68852-68863
Lai S, Shizuri Y, Yamamura S et al (1991) Three new phenolic metalolites from Penicillium species. Heterocycles 32:297-305

Lee KH, Hayaws N, Okanos M et al (1982) Lasiodiplodin, a potent antileukemic macrolide from Euphorbia splendenfs. Phytochemistry 21:1119-1121

Lee IK, Yun BS, Cho SM et al (1996) Betulinans A and B, two benzoquinone compounds from Lenzites betulina. J Nat Prod 59:1090-1092

Li W, Li XB, Lou HX (2018) Structural and biological diversity of natural p-terphenyls. J Asian Nat Prod Res 20:1-13

Liao HX, Zheng CJ, Huang GL et al (2019) Bioactive polyketide derivatives from the mangrove-derived fungus Daldinia eschscholtzii HJ004. J Nat Prod 82:2211-2219

Lin Z, Zhu T, Fang Y et al (2008) Polyketides from Penicillium sp. JP-1, an endophytic fungus associated with the mangrove plant Aegiceras corniculatum. Phytochemistry 69:1273-1278

Liu JK, Hu L, Dong ZJ et al (2004) DPPH radical scavenging activity of ten natural p-terphenyl derivatives obtained from three edible mushrooms indigenous to China. Chem Biodivers 1:601-605

Liu Y, Yang Q, Xia G et al (2015a) Polyketides with $\alpha$-glucosidase inhibitory activity from a mangrove endophytic fungus, Penicillium sp. HN29-3B1. J Nat Prod 78:1816-1822

Liu Z, Xia G, Chen S et al (2014a) Eurothiocin A and B, sulfurcontaining benzofurans from a soft coral-derived fungus Eurotium rubrum SH-823. Mar Drugs 12:3669-3680

Liu Y, Xia G, Li H et al (2014b) Vermistatin derivatives with $\alpha$ glucosidase inhibitory activity from the mangrove endophytic fungus Penicillium sp. HN29-3B1. Planta Med 80:912-917

Liu DS, Yan L, Ma LY et al (2015b) Diphenyl derivatives from coastal saline soil fungus Aspergillus iizukae. Arch Pharmacol Res 38:1038-1043

Liu Z, Chen S, Qiu P et al (2017) (+)- and (-)-Ascomlactone A: a pair of novel dimeric polyketides from a mangrove endophytic fungus Ascomycota sp. SK2YWS-LOrg. Biomol Chem 15:10276-10280

Liu M, Qi C, Sun W et al (2018a) $\alpha$-Glucosidase inhibitors from the coral-associated fungus Aspergillus terreus. Front Chem 6:422

Liu M, Sun W, Wang J et al (2018b) Bioactive secondary metabolites from the marine-associated fungus Aspergillus terreus. Bioorg Chem 80:525-530

Lopéz D, Cherigo L, Mejia LC et al (2019) $\alpha$-Glucosidase inhibitors from a mangrove associated fungus, Zasmidium sp. strain EM5-10. BMC Chem 13:22

Ma K, Han J, Bao L et al (2014) Two sarcoviolins with antioxidative and $\alpha$-glucosidase inhibitory activity from the edible mushroom sarcodon leucopus collected in Tibet. J Nat Prod 77:942-947

Mahmood ZA, Ahmed SW, Azhar I et al (2010) Bioactive alkaloids produced by fungi. I. Updates on alkaloids from the species of the genera Boletus, Fusarium and psilocybe. Pak J Pharm Sci 23(3):349-357

Nagasawa I, Kaneko A, Suzuki T et al (2014) Potential antiangiogenesis effects of p-terphenyl compounds from Polyozellus multiplex. J Nat Prod 77:963-968 
Natori S, Sato F, Udagawa S (1965) Anthraquinone metabolites of Talaromyces avellanens (Thom et Turreson), C.R. Benjamin and Preussia multispora (Saito et Minoura) Cain. Chem Pharm Bull 13:385-389

Nishida H, Tomoda H, Cao J et al (1991) Purpactins, new inhibitors of acyl-CoA: cholesterol acyltransferase produced by Penicillium purpurogenum. II. Structure elucidation of purpactins A. B and C. J Antibiot 44:144-151

Nong XH, Zhang XY, Xu XY et al (2014) Alkaloids from Xylariaceae sp., a marine-derived fungus. Nat Prod Commun 9(4):467-468

Ola A, Thomy D, Lai D et al (2013) Inducing secondary metabolite production by the endophytic fungus Fusarium tricinctum through coculture with Bacillus subtilis. J Nat Prod 76:2094-2099

Osmanova N, Schultze W, Ayoub N (2010) Azaphilones: a class of fungal metabolites with diversebiological activities. Phytochem Rev 9:315-342

Pervaiz A, Khan R, Anwar F et al (2016) Alkaloids: an emerging antibacterial modality against methicillin resistant Staphylococcus aureus. Curr Pharm Des 22(28):4420-4429

Pochet L, Frédérick R, Masereel B (2004) Coumarin and isocoumarin as serine protease inhibitors. Curr Pharm Des 10:3781-3796

Quang DN, Hashimoto T, Nukada M et al (2003) Thelephantins A, B and C: three benzoyl p-terphenyl derivatives from the inedible mushroom Thelephora aurantiotincta. Phytochemistry 62:109-113

Rehman S, Khan H (2016) Advances in antioxidant potential of natural alkaloids. Curr Bioact Comp 13:101-108

Ren J, Ding SS, Zhu A et al (2017) Bioactive azaphilone derivatives from the fungus Talaromyces Aculeatus. J Nat Prod 80:2199-2203

Rivera-Chávez J, González-Andrade M, González MC et al (2013) Thielavins A, J and K: $\alpha$-Glucosidase inhibitors from MEXU 27095, an endophytic fungus from Hintonia latiflora. Phytochemistry 94:198-205

Saeed A (2016) Isocoumarins, miraculous natural products blessed with diverse pharmacological activities. Eur J Med Chem 30:290-317

Salloum RM, Jaskowiak NT, Mauceri HJ et al (2000) NM-3, an isocoumarin, increases the antitumor effects of radiotherapy without toxicity. Cancer Res 60:6958-6963

Schaefer S, Schwaiger S, Stuppner H (2017) Aristolic acid derivatives from the bark of Antidesma ghaesembilla. Planta Med 83(12/13):1097-1102

Shen W, Mao H, Huang Q et al (2015) Benzenediol lactones: a class of fungal metabolites with diverse structural features and biological activities. Eur J Med Chem 97:747-777

Shibata S, Udagawa S (1963) Metabolic products of fungi. XIX. Isolation of rugulosin from Penicillium brunneum Udagawa. Chem Pharm Bull 11:402-403

Shibata S, Shoji J, Ohta A et al (1957) Metabolic products of fungi. XI. Some observations on the occurrence of skyrin and rugulosin in mold metabolites with reference to structural relationships between penicilliopsin and skyrin. Chem Pharm Bull 5:380-383

Srivastava AK (2019) The role of fungus in bioactive compound production and nanotechnology. In: Role of plant growth promoting microorganisms in sustainable agriculture and nanotechnology. Woodhead Publishing, Cambridge, p 145
Sun HH, Barrow CJ, Sedlock DM et al (1994) Cooper R. Benzomalvins, new substance $\mathrm{P}$ inhibitors from a Penicillium sp. J Antibiot 47(5):515-522

Sun Y, Liu J, Li L et al (2018a) New butenolide derivatives from the marine sponge-derived fungus Aspergillus terreus. Bioorg Med Chem Lett 28:315-318

Sun K, Zhu G, Hao J et al (2018b) Chemical-epigenetic method to enhance the chemodiversity of the marine algicolous fungus, Aspergillus terreus OUCMDZ-2739. Tetrahedron 74:83-87

Sutherland A, Auclair K, Vederas JC (2001) Recent advances in the biosynthetic studies of lovastatin. Curr Opin Drug Discov Dev 4:229-236

Takahashi S, Kakinuma N, Iwai H et al (2000) Quinolactacins A, B and C: novel quinolone compounds from Penicillium sp. EPF-6. II. Physicochemical properties and structure elucidation. J Antibiot 53(11):1252-1256

Talontsi FM, Dittrich B, Schueffler A, et al (2013) Epicoccolides: antimicrobial and antifungal polyketides from an endophytic fungus Epicoccum sp. associated with Theobroma cacao. Eur J Org Chem 3174-3180

Tomoda H, Nishida H, Masuma R et al (1991) Purpactins, new inhibitors of acyl-CoA: cholesterol acyltransferase produced by Penicillium purpurogenum. I. Production, isolation and physico-chemical and biological properties. J Antibiot 44:136-143

Tousif MI, Shazmeen N, Riaz N et al (2014) $\alpha$-Glucosidase and lipoxygenase inhibitory derivatives of cryptosporioptide from the endophytic fungus Cryptosporiopsis sp. J Asian Nat Prod Res 16(11):1068-1073

Usman B, Sharma N, Satija S et al (2019) Developments in alpha-glucosidase inhibitors for management of type-2 diabetes: an update. Curr Pharm Des 25:2510-2525

Valle PD, Martínez AL, Figueroa M et al (2016) Alkaloids from the fungus Penicillium spathulatum as $\alpha$-glucosidase inhibitors. Planta Med 82:1286-1294

Wang C, Guo L, Hao J et al (2016) $\propto$-Glucosidase Inhibitors from the marine-derived fungus Aspergillus flavipes HN413. J Nat Prod 79:2977-2981

Wang L, Dong Y, Song H et al (2008) Screening and isolationof antibacterial activities of the fermentative extracts offreshwater fungi from Yunna Province, china. Ann Microbiol 58:579-584

Wang BT, Qi QY, Ma K et al (2014) Depside $\alpha$-glucosidase inhibitors from a culture of the mushroom Stereum hirsutum. Planta Med 80:918-924

Wang SM, Han JJ, Ma K et al (2014a) New $\boldsymbol{\alpha}$-glucosidase inhibitors with p-terphenyl skeleton from the mushroom Hydnellum concrescens. Fitoterapia 98:149-155

Wang JF, Zhou LM, Chen ST et al (2018) New chlorinated diphenyl ethers and xanthones from a deep-sea-derived fungus Penicillium chrysogenum SCSIO 41001. Fitoterapia 125:49-54

Wells JM, Cole RJ, Kirksey JW (1975) Emodin, a toxic metabolite of Aspergillus wentii isolated from weevildamaged chestnuts. Appl Microbiol 30:26-28

Wu Y, Chen Y, Huang X et al (2018) $\alpha$-Glucosidase inhibitors: diphenyl ethers and phenolic bisabolane sesquiterpenoids from the mangrove endophytic fungus Aspergillus flavus QQSG-3. Mar Drugs 16(9):307 
Xiao Z, Chen S, Cai R et al (2016) New furoisocoumarins and isocoumarins from the mangrove endophytic fungus Aspergillus sp. 085242. Beilstein J Org Chem 12:2077-2085

Xu L, He Z, Xue J et al (2010) $\beta$-Resorcylic acid lactones from a Paecilomyces fungus. J Nat Prod 73:885-889

Xu HW, Xu C, Fan Z et al (2013) A facile synthesis, antibacterial activity of pulvinone and its derivatives. Bioorg Med Chem Lett 23:737-739

Xu Y, Zhou T, Patricia EA et al (2014) Insights into the biosynthesis of 12-membered resorcylic acid lactones from heterologous production in Saccharomyces cerevisiae. Chem Biol 9:1119-1127

Yamazaki M, Maebayashi Y, Miyaki K (1971) The isolation of secalonic acid A from Aspergillus ochraceus cultured on rice. Chem Pharm Bull 19:199-201

Yang R, Li C, Lin Y et al (2006) Lactones from a brown alga endophytic fungus (No. ZZF36) from the South China Sea and their antimicrobial activities. Bioorg Med Chem Lett 16:4205-4208

Yang ZJ, Yin Y, Ge M (2015) A novel chromene with antitumor activities from fungus Phomopsis sp. Zhongguo Zhongyao Zazhi 40(4):667-671

Yaya AJG, Feumba RD, Emmanuel T et al (2014) Antioxidant activity of compounds isolated from the root woods of Erythrina droogmansiana. Int J Pharm Sci Drug Res 6(2):160-163

Yin L, Ohno T, Weichselbaum R et al (2001) The novel isocoumarin 2-(8-hydroxy-6-methoxy-1-oxo-1H-2-benzopyran-3-yl) propionic acid (NM-3) induces lethality of human carcinoma cells by generation of reactive oxygen species. Mol Cancer Ther 1:43-48

Yin Z, Zhang W, Feng F et al (2014) $\alpha$-Glucosidase inhibitors isolated from medicinal plants. Food Sci Hum Wellness 3:136-174

Ying YM, Fang CA, Yao FQ et al (2017) Bergamotane sesquiterpenes with alpha-glucosidase inhibitory activity from the plant pathogenic fungus Penicillium expansum. Chem Biodiv 14:e160018

Zhang LH, Feng BM, Zhao YQ et al (2016a) Polyketide butenolide, diphenyl ether, and benzophenone derivatives from the fungus Aspergillus flavipes PJ03-11. Bioorg Med Chem Lett 26:346-350

Zhang LH, Feng BM, Sun Y et al (2016b) Flaviphenalenones A$\mathrm{C}$, three new phenalenone derivatives from the fungus Aspergillus flavipes PJ03-11. Tetrahedron Lett 57:645-649

Zhang L, Niaz SI, Wang Z et al (2017) $\alpha$-Glucosidase inhibitory and cytotoxic botryorhodines from mangrove endophytic fungus Trichoderma sp. 307. Nat Prod Res 32:2887-2892

Zhao XR, Huo XK, Dong PP et al (2015) Inhibitory effects of highly oxygenated lanostane derivatives from the fungus ganoderma lucidum on P-glycoprotein and $\alpha$-glucosidase. J Nat Prod 78:1868-1876

Zheng CJ, Huang GL, Liao HX et al (2019) Bioactive cytosporone derivatives isolated from the mangrove-derived fungus Dothiorella sp. ML002. Bioorg Chem 85:382-385

Publisher's Note Springer Nature remains neutral with regard to jurisdictional claims in published maps and institutional affiliations. 